\title{
A systematic review of research on cheating in online exams from 2010 to 2021
}

\author{
Fakhroddin Noorbehbahani ${ }^{1}$. Azadeh Mohammadi ${ }^{1}$. \\ Mohammad Aminazadeh ${ }^{1}$
}

Received: 26 October 2021 / Accepted: 31 January 2022 / Published online: 7 March 2022

(c) The Author(s), under exclusive licence to Springer Science+Business Media, LLC, part of Springer Nature 2022

\begin{abstract}
In recent years, online learning has received more attention than ever before. One of the most challenging aspects of online education is the students' assessment since academic integrity could be violated due to various cheating behaviors in online examinations. Although a considerable number of literature reviews exist about online learning, there is no such review study to provide comprehensive insight into cheating motivations, cheating types, cheating detection, and cheating prevention in the online setting. The current study is a review of 58 publications about online cheating, published from January 2010 to February 2021. We present the categorization of the research and show topic trends in the field of online exam cheating. The study can be a valuable reference for educators and researchers working in the field of online learning to obtain a comprehensive view of cheating mitigation, detection, and prevention.
\end{abstract}

Keywords Online exam - Cheating types · Cheating detection $\cdot$ Cheating prevention $\cdot$ Cheating motivations $\cdot$ Systematic mapping review.

Fakhroddin Noorbehbahani

noorbehbahani@eng.ui.ac.ir

Azadeh Mohammadi

az.mohammadi@eng.ui.ac.ir

Mohammad Aminazadeh

m.aminazadeh@mehr.ui.ac.ir; mohammad.aminazadeh98@gmail.com

1 Faculty of Computer Engineering, University of Isfahan, Azadi square, 8174673441 Isfahan, Iran 


\section{Introduction}

Today, distance education has been transformed into online settings, and the COVID-19 pandemic has raised online learning significantly across the world. The COVID-19 enforced the closing of traditional learning all over the world, resulting in 1.5 billion students and 63 million educators shifting from face-to-face learning to online learning. This situation has revealed the strengths and weaknesses of the digital transformation of education (Valverde-Berrocoso et al., 2020).

In (Martin et al., 2020), it has been shown that the online learning publications are continuously being increased from 2009 to 2018, and one of the leading research themes is course assessment. Course assessment is very challenging in online learning due to the lack of direct control over students and educators.

For an educational institution, assessment integrity is essential because it affects institutional reputation. It is necessary to employ traditional cheating detection besides prevention methods and new digital monitoring and validation techniques to support assessment integrity in online exams (Fluck, 2019).

The study (Watson \& Sottile, 2010) has reported that students are remarkably more likely to get answers from others during online exams or quizzes compared to live (face-to-face) ones. Therefore, preserving the integrity of online exams is more challenging. There are some strategies to mitigate online exam cheating, such as getting offline (face-to-face) proctored exam, developing cheat-resistant questions (e.g., using subjective measures instead of objective measures), and lessening the exam score percentage contributing to the overall course grade.

Traditional cheating methods include, hiding notes in a pencil case, behind ruler, or clothes, writing on arms/hands, leaving the room, etc. (Curran et al., 2011). Technological advances and online learning have enhanced education, however, they also have facilitated cheating in courses (Turner \& Uludag, 2013). For instance, an examinee could use a mobile phone to text someone to get the answer. Although this would be difficult in the exam hall, some examinees could text without looking at the mobile phone. Applying scientific calculators, Mp3 players calculator, and wireless equipment such as an earphone and a microphone are other tools that facilitate cheating in offline exams (Curran et al., 2011).

Although cheating motivations in online and offline exams are not significantly different (Turner \& Uludag, 2013), detecting and mitigating online cheating could be more intricate. This is because, in addition to traditional cheating methods that also could be exploited in online exam cheating, there exist various technologies and tools that could be applied for cheating in online exams more easily. For example, using remote desktop and share screen, searching for solutions on Internet, using social networks, etc.

Cheating in an online setting is more convenient than a traditional offline exam. Accordingly, detecting and preventing online cheating is critical for online assessment. Therefore, this issue is one of the biggest challenges that MOOC (Massive Open Online Courses) summative assessment faces.

Recent researches imply that a critical issue in online education is academic dishonesty and cheating. Today, paid services exist that impersonate students in online 
courses to ensure their identity. In recent years, proctoring technologies such as identity authentication, keystroke recognition, and webcam proctoring will be extended to secure online exams (Xiong \& Suen, 2018). Apart from direct proctoring, there are some techniques such as controlling the browser, limiting exam time, randomizing questions and choices, etc. However, it seems cheating in online courses is pretty common (Dendir \& Maxwell, 2020).

Although one of the most critical challenges in online learning is to mitigate and handle cheating, there is no comprehensive literature review and classification in this field. Hence, in this paper, we present a systematic mapping review of researches in online examination cheating. The research questions are as follows:

RQ1: What are the publication trends in online cheating?

RQ2: What are the main reasons for online cheating?

RQ3: What are the cheating types in online exams?

RQ4: How can online cheating be detected?

RQ5: How can online exam cheating be prevented?

The paper is structured as follows. In Section 2, the research method is described, including study selection criteria, databases and search strategy, and study selection. Section 3 presents review results and provides the answers to research questions. Sections 4 and 5 discuss the results and conclude the paper, respectively.

\section{Method}

The current study is a literature review about cheating in online exams. A literature review identifies, selects, and synthesizes primary research studies in order to provide a picture of the topic under investigation. According to (Page et al., 2021), a record is the title or abstract (or both) of a report indexed in a database or website, and a report is a document (in paper or electronic format) supplying information about a particular study. It could be a journal article, preprint, conference abstract, study register entry, clinical study report, dissertation, unpublished manuscript, government report, or any other document providing relevant information. The current literature search has been performed based on the well-established PRISMA principles (Page et al., 2021).

\subsection{Inclusion and exclusion criteria}

The main criteria for the articles considered in the current review are as follows. Inclusion criteria:

- Researches should be written in English.

- Records should be retrieved utilizing the designed search query. 
- Studies should be published between January 2010 and February 2021.

- In cases where several papers reported the same study, only the most recent ones were included (i.e., theses and papers extracted from theses, extended version of papers published in journals).

Exclusion criteria:

- Papers merely related to methods applicable to traditional cheating types, detection, and prevention are eliminated.

- Studies not related to research questions are ignored.

- Articles only related to cyber-attacks to online exam systems are excluded.

- Low-quality researches are discarded (i.e., studies published by non-reputable publishers without peer review, too short review time, and so on, studies with poor theoretical background, experimental evaluation, or structure).

\subsection{Databases and search strategy}

We applied a wide range of databases as our primary source, including Google Scholar, Web of Science, and Scopus. We also added the publications which had cited the extracted records. Records were searched using the following search terms for the title, keywords, and abstract sections.

(Cheat OR e-Cheating OR Fraud OR Dishonesty OR Anti-cheating OR Cheatresistant OR Abnormal behavior OR Misconduct OR Integrity OR Plagiarism) AND

(Electronic OR Online OR Digital OR Virtual OR Cyber OR Academic) AND

(Exam OR e-Exam OR Course OR e-Course OR Assessment OR e-Assessment OR Test OR e-Test OR Environment OR e-Environment) AND

(Prevent OR Detect OR Mitigate OR Reduce OR Minimize OR Monitor OR Proctor OR Reason OR Motivation OR Type OR Deter OR Control).

\subsection{Study selection}

The search result included 289 records, 26 of which were duplicated, and so they were deleted. From 263 screened records, 54 records were excluded by examining either the title or the abstract. In the next step, 12 reports were eliminated because they were not retrieved because were not accessible. Furthermore, after full-text eligibility checking, 144 reports have been excluded according to the inclusion and exclusion criteria as mentioned earlier.

This resulted in 53 reports that along with 5 other reports (obtained from citation searching and assessed for eligibility), were finally selected for literature review about online cheating. The flow of information through different phases of the review is presented in the PRISMA flow diagram depicted in Fig. 1. 


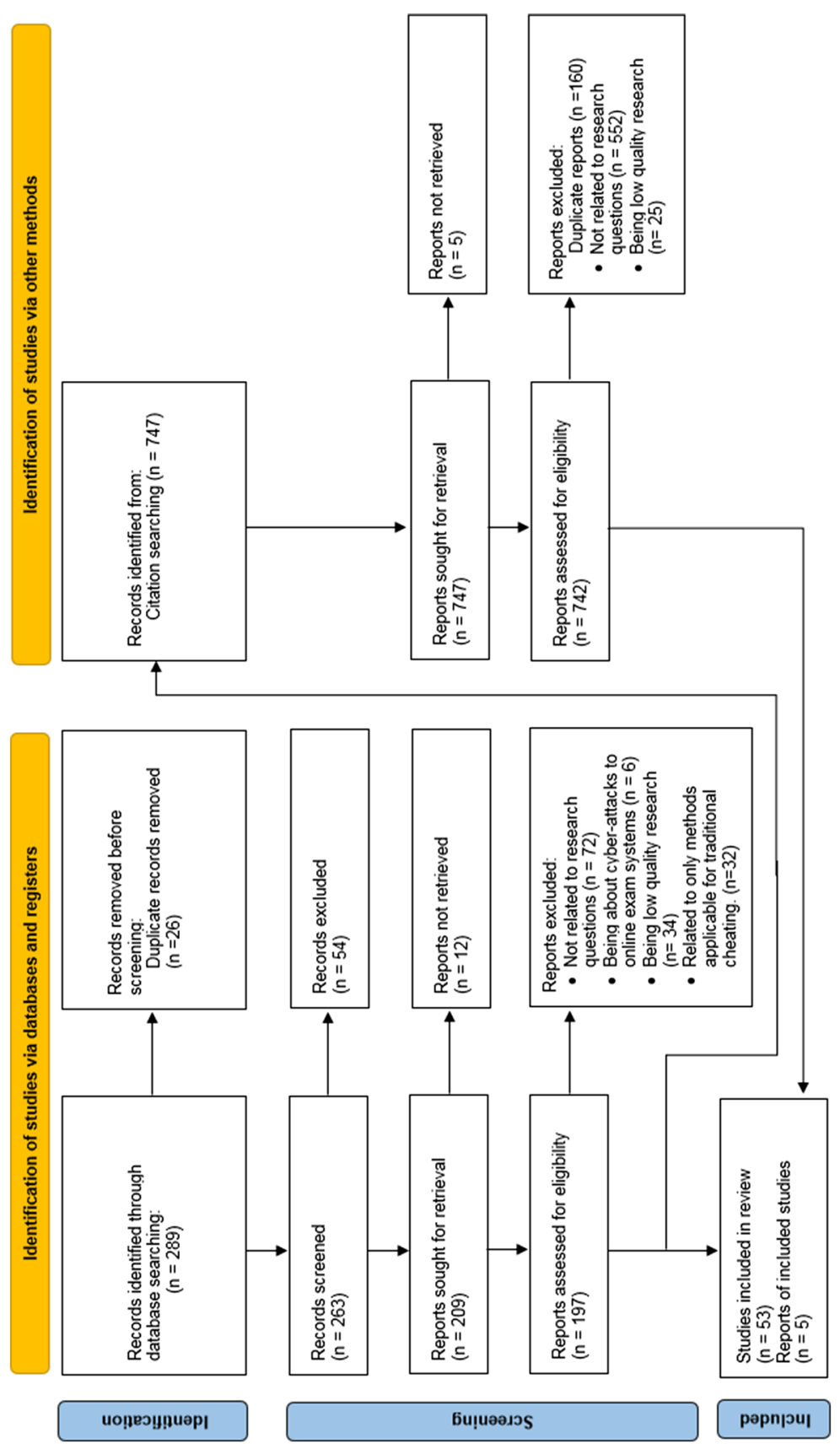

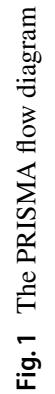


After selecting 58 studies, three domain experts were asked to assign a Credibility Score (CS) to each study. After evaluation of each study, experts agreed on a credibility score ranging from 0 to 5 based on the following criteria: publisher credibility, number of citations per year, theoretical and experimental quality, and organization and structure. CS statistics are as follows: mean $=3.81, S D=0.79$, $\min =$ 2.5, $\max =5$.

A summary of online cheating research papers and their study themes is presented in Table 1. (Appendix 1.)

\section{Results}

Several findings emerged as a result of the research synthesis of the selected fiftyeight records on online cheating. The selected studies were categorized into four main topics, namely Cheating reasons, Cheating types, Cheating detection, and Cheating prevention, as shown in Fig. 2. All subsequent classifications reported in this paper have been provided by the authors. The studies under every four main topics are investigated by three experts, and a list of items is extracted for each category. Notably, some studies were corresponded to multiple main topics. Next, several brainstorming sessions have been conducted to classify each main topic further. To extract the classifications, the XMind tool has been employed, which is a professional and popular mind mapping software.

In the following sub-sections, the detailed analysis of the review results is described according to the five research questions we defined to drive the research.

\subsection{Publication trends}

In Fig. 3, the number of publications per year is displayed (in this study, the final publication date is applied). In 2017, the greatest number of studies corresponding to the conducted review have been published. As shown in Fig. 4, the dominant publication type is journal papers with $53 \%$ of the total publications. In terms of the average citations of the selected studies regarding their classes, the maximum average citations belong to the journal papers with an average citation of 19.65 (see Fig. 5).

There are 747 works cite the selected studies related to the review. As displayed in Fig. 6, the greatest and lowest shares of the total citations pertain to the journal articles and the theses, respectively. The number of publications per research theme is shown in Fig. 7. The cheating prevention and detection themes are the most prevalent research themes in online cheating. In the following four subsections, the studies under each of the four research themes are described and classified thoroughly.

\subsection{Cheating reasons}

The primary reason for cheating is that examinees feel the rewards outweigh the risks (Lancaster \& Clarke, 2017). There exists a wide variety of reasons why 


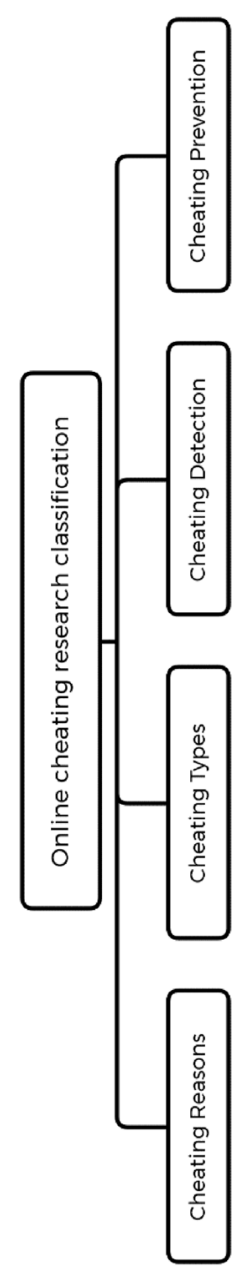

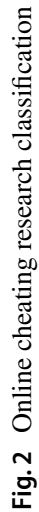




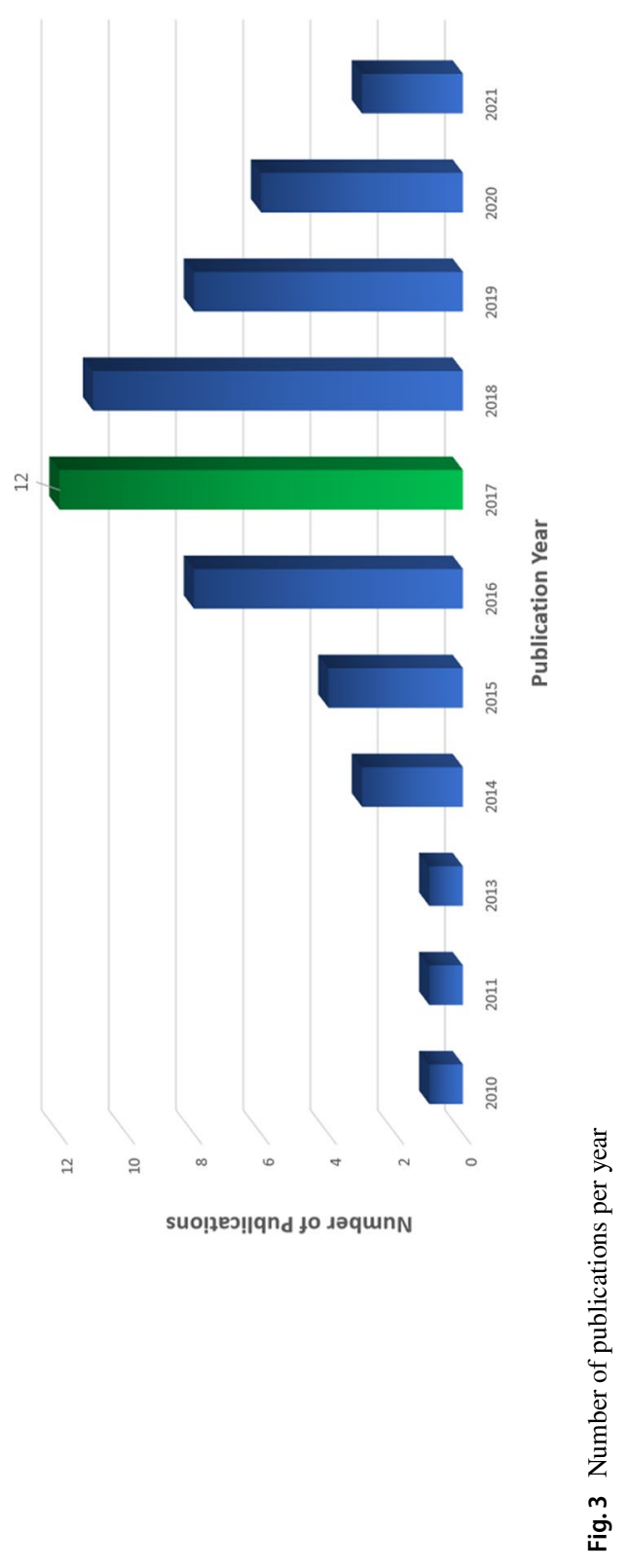




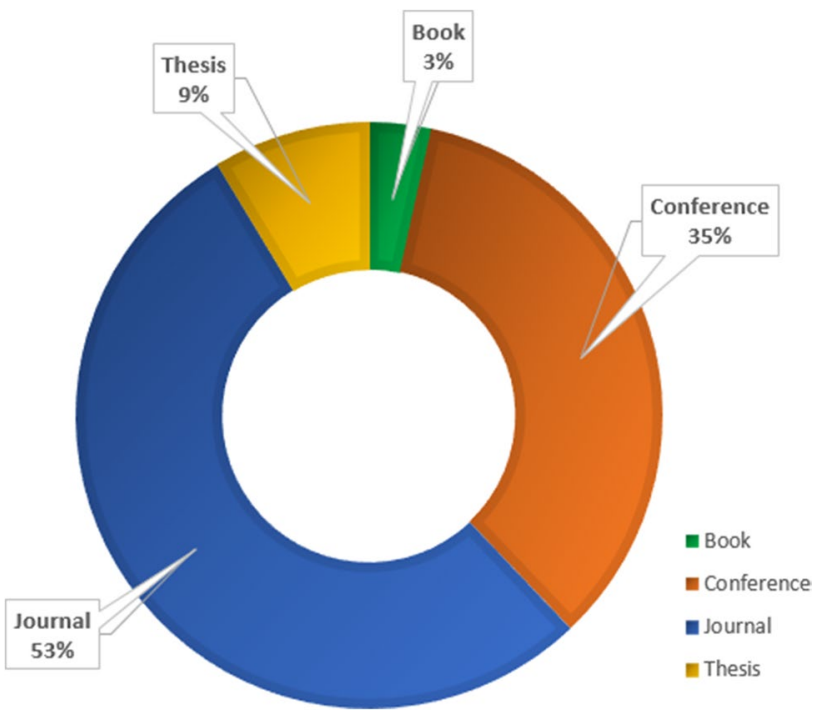

Fig. 4 Distribution of publication per types

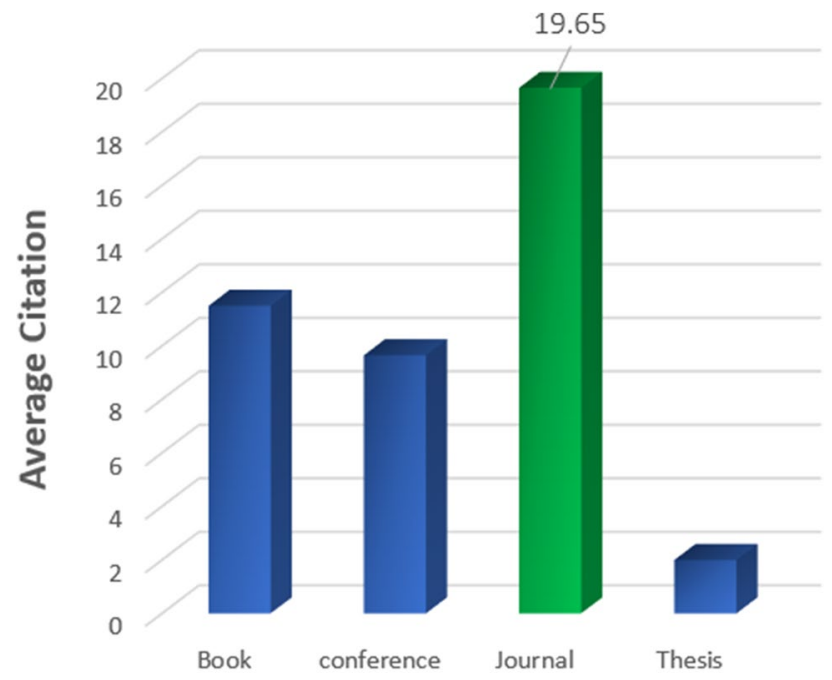

\section{Publication Type}

Fig. 5 Average citation per publication type

candidates decide to commit cheating, still, they could be categorized into four general reasons, namely Teacher-related, Institutional, Internal, and Environmental reasons. The complete classification of the cheating reasons is displayed in Fig. 8, which is described in the following sections. 


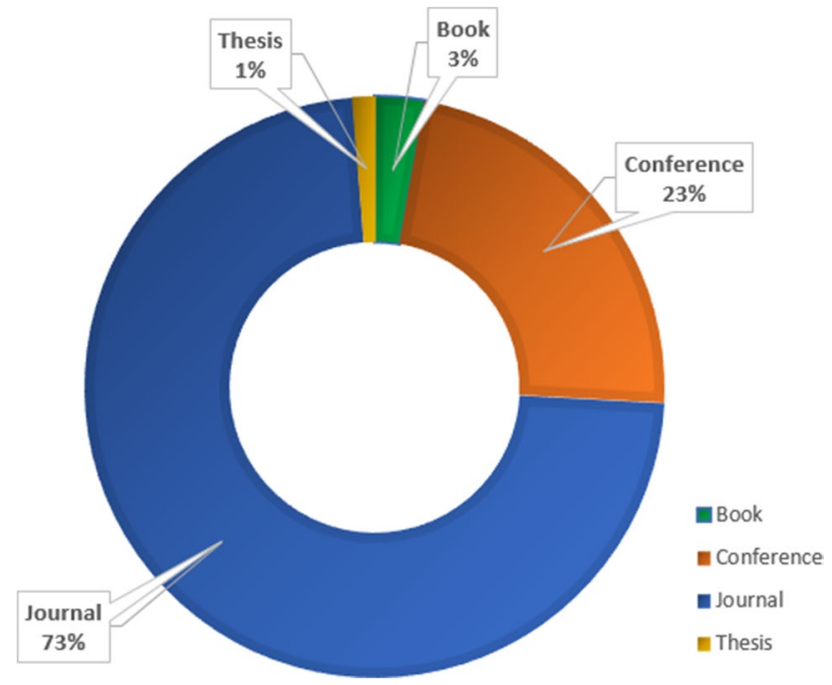

Fig. 6 Distribution of publications according to citations

\subsubsection{Teacher-related reasons}

All the reasons related to the teacher or the course instructor are put into this category. Maeda (2019), has observed that the student's relationship with the teacher has crucial influences on academic integrity. Teachers' unethical behaviors, such as favoring those who have bribed over those who have not, or favoring the students who participated in private tutoring sessions, motivate the oppressed students to cheat. The author also found that teachers' low interest in students' depth of learning, which also results in a poor pedagogical style, could be an important reason that motivates students to participate in any kind of unethical behavior (Maeda, 2019).

Course difficulty could motivate the examinees to cheat. Some students blamed their teachers for complicated and complex course materials. In some specific cases, this reason could be a consequence of students' lack of perseverance. They find cheating as a way to relieve these difficulties (Amigud \& Lancaster, 2019).

As a result of distributed learning with online courses and examinations, Moten et al. (2013), have expressed that students feel isolated in an online environment. They often become frustrated when they do not get the help they immediately need, for instance, the night before an exam. This situation is closely dependent on the presence time of the teacher in online communication environments.

Some teachers restrain from punishing the cheaters appropriately due to ethical issues. This could be due to the sympathy of some teachers with cheaters. After listening to the cheater's excuses and justifications, the teacher might give them a second chance. Sometimes, teachers are worried about the consequences 


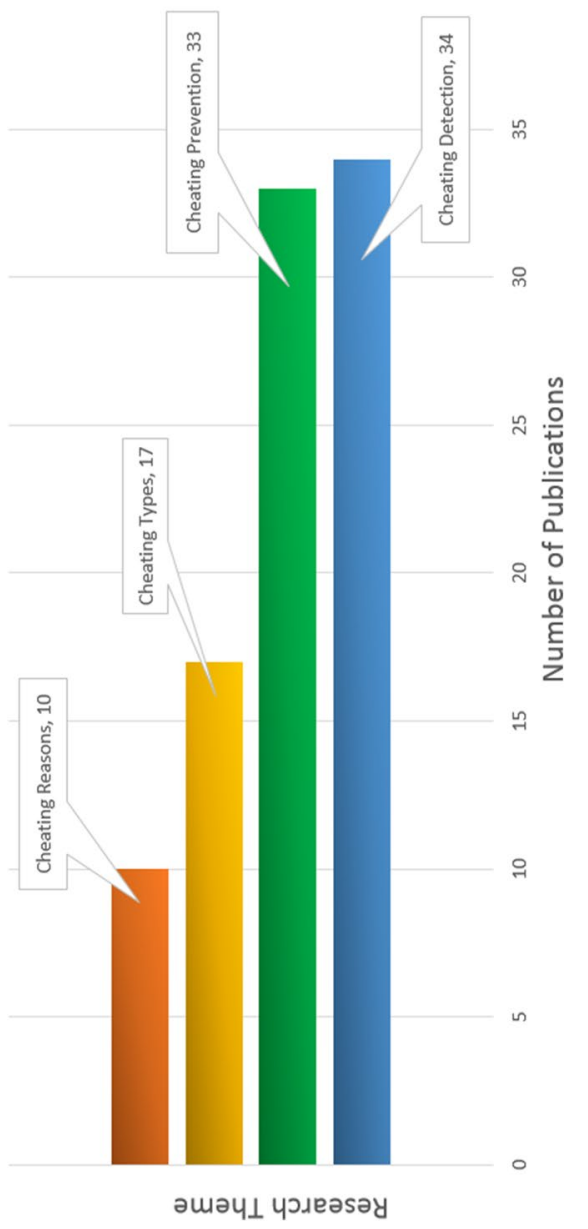

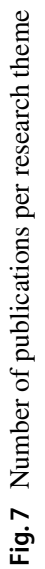




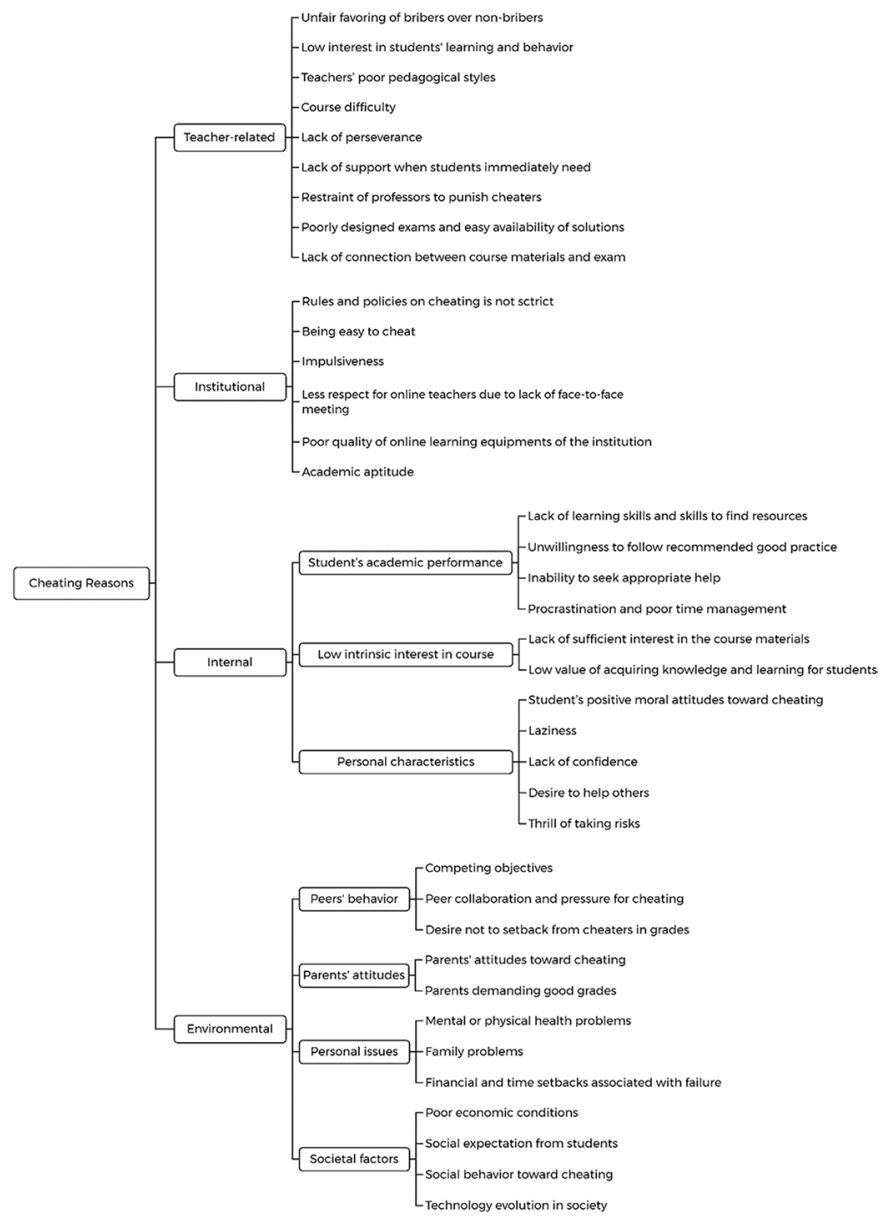

Fig. 8 Cheating reasons

of punishments and the corresponding pressures that cheaters experience, hence they don't punish the cheater or the punishment is too mellow.

This increases the students' courage to cheat during online exams due to decreased risk of being punished after being caught and implies that cheating penalties are insignificant over the long run (Topîrceanu, 2017).

Exam design is one of the most important contributing factors that motivates examinees to cheat in the exam. Weakly designed exams such as similar multiplequestions for every examinee or easy accessibility of solutions over the web, can make it easy to cheat. On the other hand, questions being too complex and irrelevant to course materials, forces students to commit cheating during online exams (Srikanth \& Asmatulu, 2014). 


\subsubsection{Institutional reasons}

In (Maeda, 2019), it is observed that the rules and policies of the institution are directly related to the number of unethical behaviors occurrences. It is found that institutions with stricter regulations and better commitment to strengthening academic integrity, face much less cheating behavior between their students. Institutional policies not only create an anti-cheating atmosphere, but also makes dishonest academic behaviors challenging to take place. Also, Backman (2019) emphasizes that if it becomes easy for students to cheat, they will cheat.

Impulsiveness is a crucial reason why students try to cheat during online examinations. They feel isolated and disconnected, so they may imagine they won't get caught or the instructor does not care if they commit academic dishonesty. Unethical behaviors have a direct relationship with the student's impulsiveness (Moten et al., 2013).

Moreover, in an isolated environment, due to the lack of face-to-face communications with teachers, students have much less respect for their teachers that leads to increasing misbehaviors. That is why teachers should personalize the online environment for students by calling their names or listening to their voices, so that online classes become more engaging and interactive for students (Moten et al., 2013).

Dobrovska (2017), expressed that the poor quality of the institution's online learning system discourages students from learning the course materials, and makes it difficult for them to learn, hence, they are more motivated to cheat.

Academic aptitude is one of the most important and underrated reasons leading students to commit misbehaviors. It means educational institutions don't discriminate between students and ignore their unique abilities, skills, and different levels of preparedness for a specific task. This makes unprepared students feel frustrated about that particular task or course, which leads them to seek help from more talented and prepared students in that specific context (Amigud \& Lancaster, 2019).

\subsubsection{Internal reasons}

Another category of cheating reasons is internal motivators. The motivators over which the candidate has complete control, including intrinsic factors, personality and psychological characteristics, lie in this category. The internal reasons are divided into three subcategories as follows.

Student's academic performance One significant internal factor is the student's academic performance. There are several reasons that could result in poor academic performance as follows: lack of learning and skills to find resources, students unwillingness to follow recommended practices, inability to seek appropriate help, procrastination, poor time management (Dobrovska, 2017), and lack of confidence in their ability to learn course materials (Norris, 2019).

Low intrinsic interest in the course materials Low intrinsic interest in the course is another reason mentioned in (Dobrovska, 2017), which could be caused by a lack of 
sufficient interest in course materials and subjects or the mindset that these materials and knowledge are unnecessary and unimportant for future life (Norris, 2019).

Personal characteristics There is a strong relationship between students' moral attitudes toward cheating and their level of participation in academic misbehaviors (Maeda, 2019). Therefore, conscientious belief is considered as an internal reason stopping students from unethical behaviors. However, it has been shown that religious beliefs do not necessarily lower cheating behaviors (Srikanth \& Asmatulu, 2014).

Other reasons included in studies are student's laziness for sufficient home preparation before the exam (Dobrovska, 2017), competition with others and the desire to get ahead (Amigud \& Lancaster, 2019), desire to help other peers (Moten et al., 2013) and the student's thrill of taking risk (Hylton et al., 2016).

\subsubsection{Environmental reasons}

The reasons mentioned in this section highly depend on the atmosphere and type of environment a student is in, either during the online exam or beforehand in social media or communication with people. We put these reasons in four major categories: Peers' behavior, Parents' attitudes, Personal issues and, Social factors.

Peers' behavior Peers could influence individuals in a manner that their cheating motivations are increased. In an academic environment, however, it is primarily because of the competing objectives, such as the desire to get ahead in scores. This depends on the amount of competition in the academic environment (Amigud \& Lancaster, 2019).

Experimental research among Cambodian students, has figured out that being among a group of cheaters, psychologically drives the students to repeat their peers' actions and commit cheating. In addition, there is high pressure on those who do not collaborate with peers, or reject participating in their group work. It is found that they are blamed for being odd and unkind (Maeda, 2019).

According to (Srikanth \& Asmatulu, 2014), being in an environment where peers' cheating remains undetected, gives this kind of feeling to non-cheaters that they are setting back in scores and are unfairly disadvantaged compared to those cheaters.

Parents' attitude Parents' acceptance of cheating behaviors, massively affects the student's mindset toward these behaviors. As expressed in (Maeda, 2019), parents' behaviors toward their child's cheating, vary from complete unacceptance to active involvement and support. Another reason related to parents' attitudes is putting their children under pressure to achieve good or higher than average grades (Backman, 2019). 
Personal issues Personal issues could be mental and physical health problems (Amigud \& Lancaster, 2019), problems within the family (e.g., parents arguing, separation and divorce, etc.), and fear of failure in exams and its further consequences like financial and time setbacks (Hylton et al., 2016).

Societal factors Poor economic conditions and the development level of a country are examples of societal factors affecting students' motivation to cheat and achieve academic success (Maeda, 2019).

Countries with various cultures, social expectancies, and people's attitudes have different behaviors regarding academic performance. In some countries, academic performance and grades are known to be crucial for success in life, whereas, in other countries, academic performance is relatively low valued. This range of different expectations from students leads to various social beliefs and behaviors toward cheating (Maeda, 2019). In research presented in (Holden et al., 2020), it is shown that a primary reason could be the existence of a cheating culture. Some students may cheat because they desire to portray a better image of themselves to their society (Norris, 2019). Another societal factor influencing cheating behaviors is the technology evolution that strengthens cheating motivation (Maeda, 2019). This is because technology brings about increased access to cheating resources. The evolution of technology, specifically search engines and social media, makes it easier for students to cheat.

\subsection{Cheating types and facilitators}

To mitigate cheating behaviors effectively and efficiently, cheating methodologies, types, and facilitators should be known. Cheating is performed either individually or by the cooperation of others (called group cheating). Figure 9 displays the complete classification of cheating types.

\subsubsection{Individual cheating}

Individual cheating is carried out without any assistance from any person. This type of cheating could be categorized as using forbidden materials and other types are described as follows.

Using forbidden materials Individual cheating can occur by using forbidden materials during the exam, such as looking at a textbook or a cheat sheet (Fontaine et al., 2020), (Holden et al., 2020), searching the web, using offline electronic resources such as images, voices, etc. (Korman, 2010), (Holden et al., 2020), or even using objects in the exam room to hide notes.

Other types Other types of individual cheating include accessing the questions and solutions before the exam, which Korman (2010) refers to as "unauthorized 


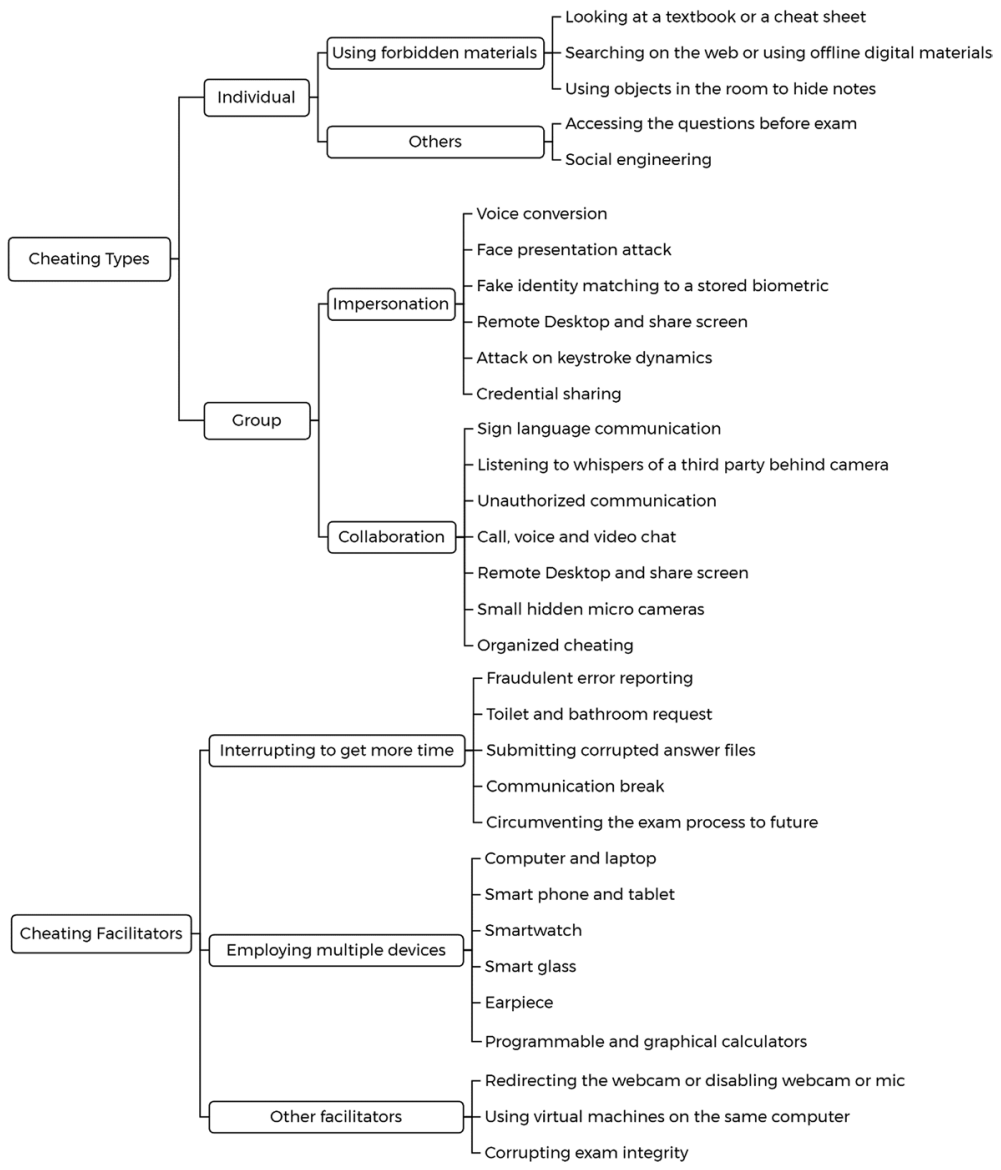

Fig. 9 Cheating types

intelligence". Another dishonest behavior is social engineering, which is grade negotiation with the teacher through fake facts and exploiting personal sympathy.

\subsubsection{Group cheating}

Cheating methods through cooperation with others could be categorized as Impersonation, and Collaboration types.

Impersonation Impersonation means employing someone to take the exam for the examinee, either the whole exam or some parts of it (Korman, 2010), (Holden et al., 2020). It can occur in forms of voice conversion, face presentation attack and face impersonation, fake identity matching to a stored biometric, and attack on the keystroke dynamics (Chirumamilla \& Sindre, 2019). These are attacks on the 
biometric system to bypass the authentication mechanisms. The other impersonation techniques include remote desktop control by a third party (Kasliwal, 2015), (Gruenigen et al., 2018), sharing the screen with a third party (Gruenigen et al., 2018), (Bawarith, 2017), and credential sharing, which is impersonation via shared username and password of an academic account or LMS (Learning Management System) (Dobrovska, 2017).

Collaboration Collaboration is defined as getting any kind of help from others to answer the exam questions. It could be in the form of sign language communications that come in numerous forms, such as foot-tapping, pencil or any object dropping during the proctored exam, abnormal coughing, or suspicious actions (Srikanth \& Asmatulu, 2014).

Listening to a third party's whispers behind the camera (Chirumamilla \& Sindre, 2019), any type of communication which is unauthorized such as sending or receiving messages, or voice and video calls (Korman, 2010), are also considered as collaborative cheating.

Other cheating methods in this category are remote desktop control (Kasliwal, 2015) and sharing the screen with others to collaborate with others about questions (Gruenigen et al., 2018), applying small hidden micro cameras to capture images and record videos for sharing with other peers (Bawarith, 2017), and finally, organizational cheating which is a result of institution's personnel corruption (Korman, 2010).

The last one, as Korman (2010) showed, can take place when personnel help candidates to cheat. Changing the exam grade or exam answers after the exam (exam integrity corruption), giving the solutions to the candidate during the exam, or just bribing the proctor not to report the cheating or not to punish after being caught (Kigwana \& Venter, 2016) are instances of organized cheating.

Contract work is a type of collaboration that means doing work with the help of someone else under the obligations of a contract. Contract workers may provide some or all of the exam answers. In this case, sometimes impersonating the student through the whole academic course is reported (Chirumamilla \& Sindre, 2019).

\subsubsection{Cheating facilitators}

Methods discussed here act as cheating facilitators to support the process of cheating. In other words, these facilitators can be applied to perform any kind of cheating. A study presented in (Peytcheva-Forsyth et al., 2018), indicates that technology in general, is the leading facilitator of cheating practices. Cheating facilitators are classified as shown in Fig. 10.

Three different methodologies are used by students to facilitate cheating, either individually or in a group, described as follows. 


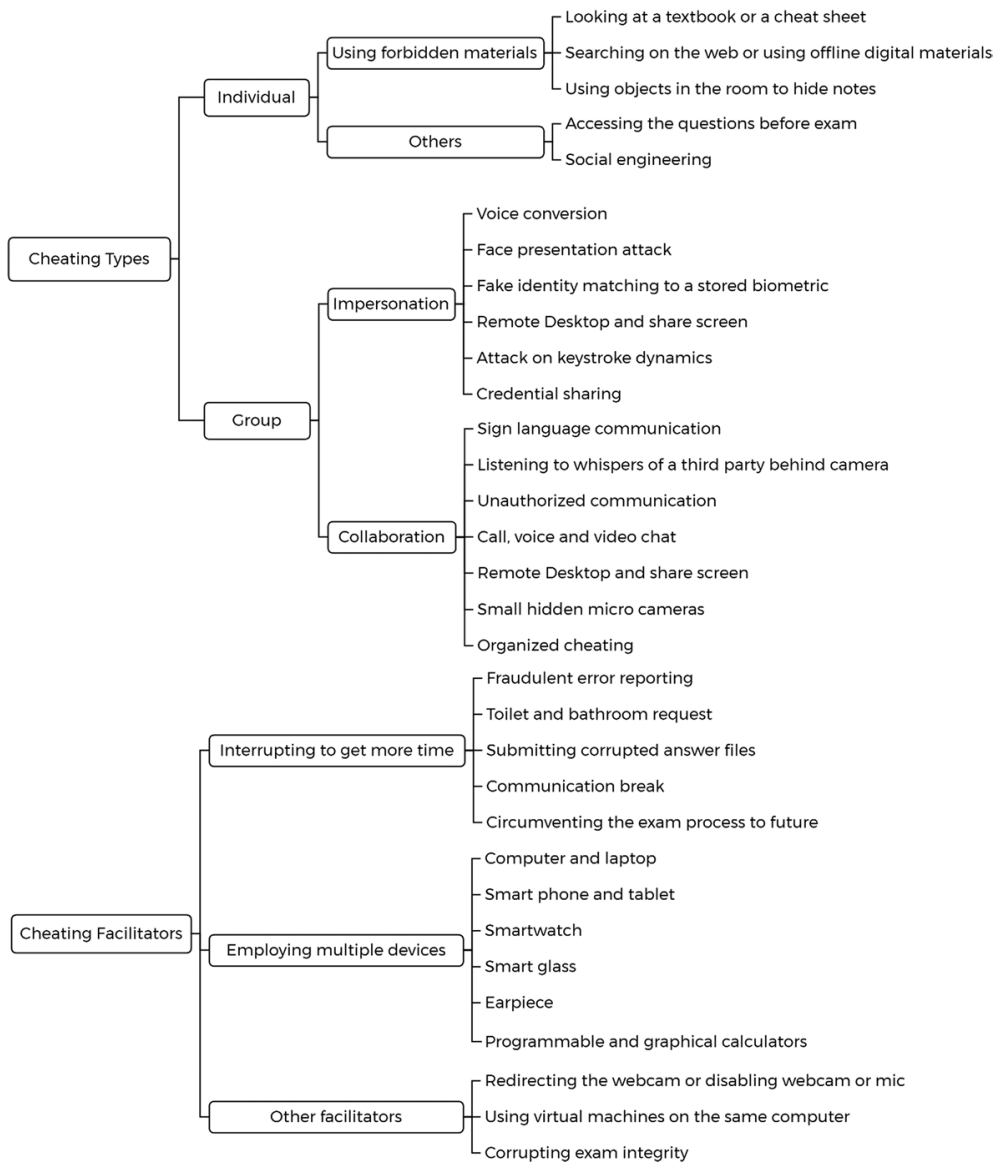

Fig. 10 Cheating facilitators

Interrupting to get more time Sometimes examinees try to buy more time to work more on the exam answers. For instance, the examinee may report an error about the exam system or exam proctoring software to convince the teacher to restart the exam session. This enables the candidate to get more time for cheating and finding the solutions during this interval when the session is closed (Motenet al., 2013). Another interruption method is to submit corrupted answer files by the candidate. In this case, the teacher reports that the files were corrupted and asks the candidate to resubmit the answer files. Most of the time, during the first submission and the second one, there exists at least one day, which implies the candidate gets at least one more day to answer the exam questions (Moten et al., 2013).

Other more classical methods to interrupt are toilet requests during the exam (Chirumamilla \& Sindre, 2019), communication break and delay in answering 
oral exam right after a question is asked (Chirumamilla \& Sindre, 2019), circumventing the exam process at a specific time with different excuses, and postponing taking the exam (Fontaine et al., 2020), (Korman, 2010). By deferring taking the exam, students can buy more time to become more prepared, either by studying more, or getting access to the exam questions and solutions.

Employing multiple devices In proctored exams, either by a camera or software, students try to use multiple devices and answer the questions with the primary one while cheating via the secondary device. Several types of devices could be employed as the second device, such as computers and laptops (Moten et al., 2013), smartwatches (Wong et al., 2017), smart glasses such as Google glasses (Srikanth \& Asmatulu, 2014), smartphones and tablets (Korman, 2010), programmable and graphical calculators to store notes and formulas (Kigwana \& Venter, 2016), and tiny earpieces for remote voice support during the exam (Bawarith, 2017).

Other facilitators Redirecting the webcam to hide something from its field of view (Sabbah, 2017), (Srikanth \& Asmatulu, 2014), or disabling the webcam or microphone completely (Srikanth \& Asmatulu, 2014) are other tricks used to facilitate cheating.

By using virtual machines on a computer, the user can run a virtual operating system on the primary one. This technique would hide the activities done on the second operating system from the software or the human proctoring the primary operating system. (Kasliwal, 2015).

Corrupting the exam system's integrity to change the exam results after being held (e.g., changing the scores or answers after the examination) is another notable case (Korman, 2010). Lastly, in (Parks et al., 2018), the authors have investigated that social media and channels operating on them could act as cheating facilitation environments.

\subsection{Cheating detection}

Cheating detection methods can be categorized into during the exam and after the exam detection methods. Further classification of the cheating detection methods is presented in Fig. 11.

\subsubsection{Cheating detection during the exam}

To ensure academic integrity in online examinations, it is essential to detect cheating during the exam. Cheating detection can be partitioned into two main categories, namely, continuous authentication and online proctoring. Continuous authentication methods verify the identity of test-takers, and online proctoring monitors the examinees to detect any misbehavior during the exam. In the following, we will mention different techniques in each category. 


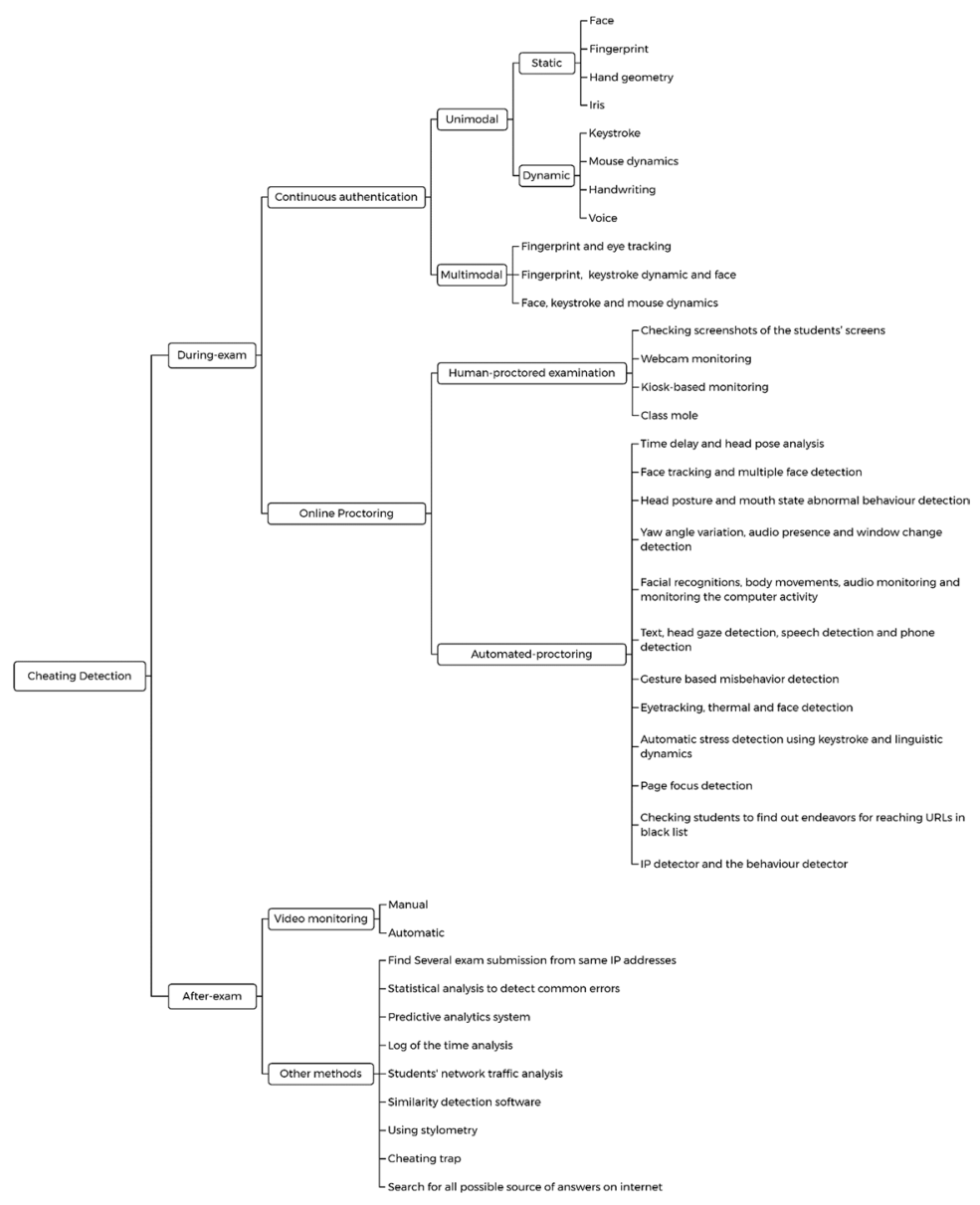

Fig. 11 Cheating detection

Continuous authentication One of the main types of cheating is impersonating. Therefore, it is essential to authenticate students before exam registration and prevent unauthorized candidates from taking the examination. In addition, it is necessary to validate the identity of the test-taker during the exam continuously. The continuous authentication systems are mainly based on biometric or behaviometric modalities and can be categorized into unimodal and multimodal schemes.

Unimodal authentication is the automatic recognition and identification of candidates using a unique characteristic. This characteristic could be either static (physiological) such as the face, fingerprint, hand geometry, and iris, or could be dynamic (behavioral) such as voice, handwriting, keystroke, and mouse dynamics (Chirumamilla \& Sindre, 2019). 
As a unimodal authentication system, Arnautovski (2019) designed a face recognition system, which captures the image of the test-taker at random time intervals. The facial recognition module continuously verifies the examinee's identity by comparing captured images to the image from the exam registration process. In (Aisyah et al., 2018), an Android-based online exam application is implemented that takes photos of the examinee with random intervals and a webbased application lets the admin or supervisor of examination validate pictures of participants. In addition, Idemudia et al. (2016) proposed a system that tracks and detects faces continuously to verify the candidates. If the authentication failure remains for more than a few seconds, the system will stop the examination.

In (Sabbah, 2017), a scheme called ISEEU is proposed, in which each examinee's session is streamed using a webcam. A proctor monitors the video screens and can generate alerts when any suspicious action is detected. He et al. (2018) proposed an anti-ghostwriter system using face recognition methods. The ghostwriter merges the student's photo and their photo to make a fake one, or they change their appearance to mislead the examiners. The experimental results in (He et al., 2018), indicate that the proposed framework can detect ghostwriters with an acceptable level of accuracy.

Since some candidates may refuse to use a camera due to privacy concerns, Bilen et al. (2020) suggested that instructors offer their students two options. An examinee can agree to use a camera during the exam. In this situation, the record will be used as evidence if they are accused of cheating. However, if the examinee doesn't accept using a camera, the instructor can claim cheating without providing evidence to the student.

In (Bawarith, 2017), the system authenticates the examinees continuously through an eye tracker. The data obtained from the eye tracker are translated into a set of pixel coordinates so that the presence or absence of eyes in different screen areas can be investigated.

Multimodal biometric authentication systems utilize different biometric or behaviometric traits simultaneously, which makes impersonating more difficult. In this regard, Bawarith et al. (2017) proposed a system that utilizes fingerprint and eye-tracking for authentication. The eye tribe tracker is used to continuously ensure that test-takers are the ones they are claiming to be. Whenever the system detects the examinee is no longer present in front of the screen, the system is locked, and the test-taker must be authenticated again via fingerprint.

In (Sabbah, 2017), a multimodal scheme called SABBAH is proposed, which adds continuous fingerprint and keystroke dynamics to the ISEEU scheme (Sabbah, 2017). In contrast to ISEEU, SABBAH uses an automatic system to detect fingerprint, keystroke, or video violations. Traore et al. (2017) proposed a system that continuously authenticates examinees using three complementary biometric technologies, i.e., face, keystroke, and mouse dynamics. In this system, test-takers are continuously authenticated in the background during the exam, and alarms are created and sent to the instructor through the proctoring panel.

Online proctoring Online proctoring is essential to promote academic integrity. Alessio et al. (2017) reported significant grade disparities in proctored versus 
un-proctored online exams. Online proctoring can be categorized into human and automated proctoring. In human proctoring, a human proctor monitors the students remotely to detect suspicious behavior. In contrast, in automated proctoring, the cheating behaviors are flagged or detected automatically by the proctoring system.

Recently, several technologies have been developed to facilitate proctoring online exams remotely. For example, Kryterion ${ }^{\mathrm{TM}}$ Live Video Monitoring and ProctorU allow users to be monitored by a human proctor via a webcam during examination (Hylton et al., 2016). In (Reisenwitz, 2020), substantial support for online proctoring is provided. The results show a significant difference between the scores of exams that were not proctored and those proctored using ProctorU software.

Some systems can capture screenshots of the candidates' PCs at random times during the examination (Migut et al., 2018). Consequently, if examinees use any forbidden resource on their computer, it will be shown to the proctor. Alessio (2018) applied video proctoring via a webcam at Miami University. The results demonstrate that students are less likely to cheat when monitored with a webcam during online testing.

In another study, kiosk-based remote online proctored examinations are compared with tests administered under a traditional proctoring environment. In kiosk-based proctoring, the test is taken on special computer kiosks located at accessible places such as libraries. The kiosks are equipped with enhanced webcams and are supervised online by a live remote proctor. The results indicated that examinees' scores obtained under online kiosk-based proctoring are comparable to examinations taken in test centers with onsite proctors (Weiner \& Hurtz, 2017).

A different approach for cheating detection is a class mole that means the instructor enrolls in students' groups under another name as a mole to detect and combat collusion. In this way, they can discover dishonest students when they discuss cheating amongst themselves (Moten et al., 2013).

Human proctoring is costly and labor-intensive. Therefore, different automated proctoring systems are proposed to monitor the students during the examination and detect unauthorized behavior. In the following, we discuss several automated methods.

Chuang et al. proposed a semi-automatic proctoring system that employs two factors, namely, time delay in answering the questions and head-pose variation, to detect suspicious behavior. Afterward, a human proctor could use more evidence to decide whether a student has cheated (Chuang et al., 2017).

Garg et al. (2020) proposed a system to detect the candidate's face using Haar Cascade Classifier and deep learning. If the examinee's face moves out of the examination frame or multiple faces are detected in the frame, the test will automatically be terminated, and the administrator will receive a notification. In (Fayyoumi \& Zarrad, 2014), a two-second candidate video is taken during the examination period. The images in the video are analyzed to verify whether the examinee is looking somewhere other than their screen. If the test-taker doesn't focus on their screen, it may indicate cheating behaviors such as looking at an adjacent PC or reading from an external source.

In (Hu et al., 2018), the proposed system uses a webcam to monitor candidates' head posture and mouth state to detect abnormal behavior. Through the rule-based 
reasoning method, the system can detect suspicious behavior such as turning heads and speaking during the online examination.

Prathish et al. (2016), developed a multimodal system for online proctoring. The system captures audios and videos of the candidates as well as their active windows. If yaw angle variations, audio presence, or window changes are detected in any time frame, it can be considered an indicator of cheating. Consequently, the captured video, audio, and system usage are fed into a rule-based inference system to detect the possibilities of misbehaviors. ProctorTrack is another automated online exam proctoring product that employs facial and audio recognition, body movements, and computer activity monitoring to detect any suspicious action during examination (Norris, 2019).

Atoum et al., (2017) developed a system that can detect a wide variety of cheating behaviors during an online exam using a webcam, wearcam, and microphone. Using wearcam makes it possible to monitor what the student observes. It helps to detect any phone or text in the testing room that is prohibited. In addition, by using the wearcam, the system can detect another form of cheating that is reading from books, notes, etc. Furthermore, the system can estimate the head gaze of the test-taker by combining the information from the webcam and wearcam. Another form of cheating is getting verbal assistance from another person in the same room, or remotely via a phone call. The system can detect this kind of cheating using the microphone and speech detection. Considering the mentioned aspects, the proposed multimedia system can perform automatic online exam proctoring.

Saba et al. (2021), developed an automatic exam activity recognition system, which monitors the body movements of the students through surveillance cameras and classifies activities into six categories using a deep learning approach. The action categories are normal performing, looking back, watching towards the front, passing gestures to other fellows, watching towards left or right, and other suspicious actions. Movement recognition based on video images is highly dependent on the quality of images. Therefore, Fan et al. (2016), employed a Microsoft Kinect device to capture the examinee's gesture. The duration and frequency of the detected action events are then used to distinguish the misbehavior from the normal behavior.

The system presented in (Mengash, 2019) includes a thermal detector attached with a surveillance camera and an eye movement tracker. When examinees intend to cheat, their body will emit a specific range of heat, and the emitted heat will trigger the camera to focus and detect the candidate's face. Then the eye tracker detects eye movements, and the system detects the cheating intentions of the test-taker. There are other biometric-based methods for cheating detection. For example, keystroke and linguistic dynamics can detect stress, which indicates suspicious behavior (Korman, 2010).

Diedenhofen and Musch (2017), developed a JavaScript application called PageFocus, which can be added to the test page and run in the background. Whenever the examinee switches to a page other than the test page, a defocusing event is registered. The script captures when and how frequently defocusing and refocusing events occur on the test page. Another method is to permit students to get to just a couple of sites that are whitelist. If the examinee tries to open a site that is not allowed (one from blacklist), the instructor will be informed through an Android application or Internet (Kasliwal, 2015).

Tiong and Lee (2021), proposed an e-cheating intelligent agent composed of two modules, namely the internet protocol (IP) detector and the behavior detector. The 
first module could monitor the examinees' IP addresses and enable the system to alert if a student changes their device or location. The second module detects abnormal behavior based on the speed of answering questions. Another method for cheating detection is comparing the IP addresses of the examinees to check whether two participants are in the same place (Bawarith, 2017).

\subsubsection{Cheating detection after the exam}

Even though different methods are employed to prevent students from cheating, some will still cheat during the examination. Consequently, a bunch of techniques is proposed to detect cheating students after the exam. This way, the reliability of online assessments will be improved. In the following, we will discuss different methods of cheating detection after the exam.

Video monitoring The University of Amsterdam has developed a system that records the student's video screen and the environment during the exam. Later a human proctor views the recording and flags and reports any suspicious behavior (Norris, 2019). Proctoring software proposed in (Alessio et al., 2017), records everything students do during the examination. After the exam, the recordings can be reviewed by the professor, teaching assistants, or employees of the proctoring vendor to identify cheating behaviors.

Human proctoring is a tedious and time-consuming process. To reduce the time and cost of proctoring, an automatic system can be employed to detect and flag suspicious events using machine learning methods. In this regard, Cote et al. (2016) proposed a system for the automatic creation of video summaries of online exams. The proposed method employs head pose estimations to model a normal and abnormal examinee's behavior. Afterward, a video summary is created from sequences of detected abnormal behavior. The video summaries can assist remote proctors in detecting cheating after the exam.

Jalali and Noorbehbahani (2017), implemented an automatic method for cheating detection using a webcam. During the exam, images are recorded every $30 \mathrm{sec}-$ onds by a webcam for each candidate. After the exam, the recorded images are compared with reference images of that student. If the difference exceeds a threshold, the image will be labeled as a cheating state.

Li et al. (2015), proposed a Massive Open Online Proctoring framework that consists of three components. First, the Automatic Cheating Detector (ACD) module uses webcam video to monitor students, and automatically flag suspected cheating behavior. Then, ambiguous cases are sent to the Peer Cheating Detector (PCD) module, which asks students to review videos of their peers. Finally, the list of suspicious cheating behaviors is forwarded to the Final Review Committee (FRC) to make the final decision.

Other methods There are various ways of cheating, and therefore, different methods are used to detect cheating after the exam. For example, one of the cheating 
behaviors is to collude and work on tests together. However, most learning management systems allow the instructor to view IP addresses. Therefore, if different students submit their assessments by the same IP address in a short time frame, it could be detected and considered as a sign of collusion (Moten et al., 2013).

In addition, statistical methods can be used to analyze student responses to assessments and detect common errors and the similarities of answers (Korman, 2010). Mott (2010) stated that the distribution of identical incorrect responses between examinee pairs is a Polya distribution. The degree of cheating for each examination will follow the skewness or third central moment of the distribution.

Predictive analytics systems implicitly collect data while the students interact with the virtual learning environment. The collected data, which include student's location, access patterns, learning progress, device characteristics, and performance, is used to predict trends and patterns of student behavior. Consequently, any unusual pattern may indicate suspicious behavior (Norris, 2019). Answering an examination takes a reasonable amount of time. Therefore, another indicator of dishonest behavior is an extremely short interval between the access time and the completion of the assessments, which can be detected by log time analysis (Moten et al., 2013).

In (Bawarith et al., 2017), an E-exam management system is proposed that classifies participants as cheating or non-cheating based on two parameters, namely the total time and the number of times the examinee is out of the screen. The focus of the test-taker is recorded using an eye tracker during the exam.

Kasliwal (Kasliwal, 2015), designed an online examination tool that captures the network traffic during the exam using a kismet server. The captured package can then be analyzed to determine the frequency of URLs accessed by students. If one of the URLs is getting accessed more frequently or very rarely, it could be considered suspicious.

To detect plagiarism in papers or essay-type questions, platforms such as DupliChecker.com ${ }^{1}$ or Turnitin.com ${ }^{2}$ can be used. These websites compute a similarity index and show all potential plagiarisms. Based on the similarity index, the instructor decides about further actions (Moten et al., 2013).

A weakness of similarity detection software is that it computes the resemblance of a submitted assessment with others' works and cannot detect an original text written by others for the student in question. Stylometry discovers this issue by checking the consistency of the delivered contents with other texts written by the same student. If the style of a text does not match with the previous works of that student, it may indicate complicity (Chirumamilla \& Sindre, 2019). Opgen-Rhein et al. (2018) presented an application that employs machine learning methods to learn the programming styles of students. This work is based on the assumption that the programming style of each student is unique, and therefore, the model can be used to verify the author of assignments.

Another way of cheating detection is using a cheating trap, which means creating websites that could be found when the students search for answers. The solutions in

\footnotetext{
1 http://www.duplichecker.com

${ }^{2}$ http://www.turnitin.com
} 


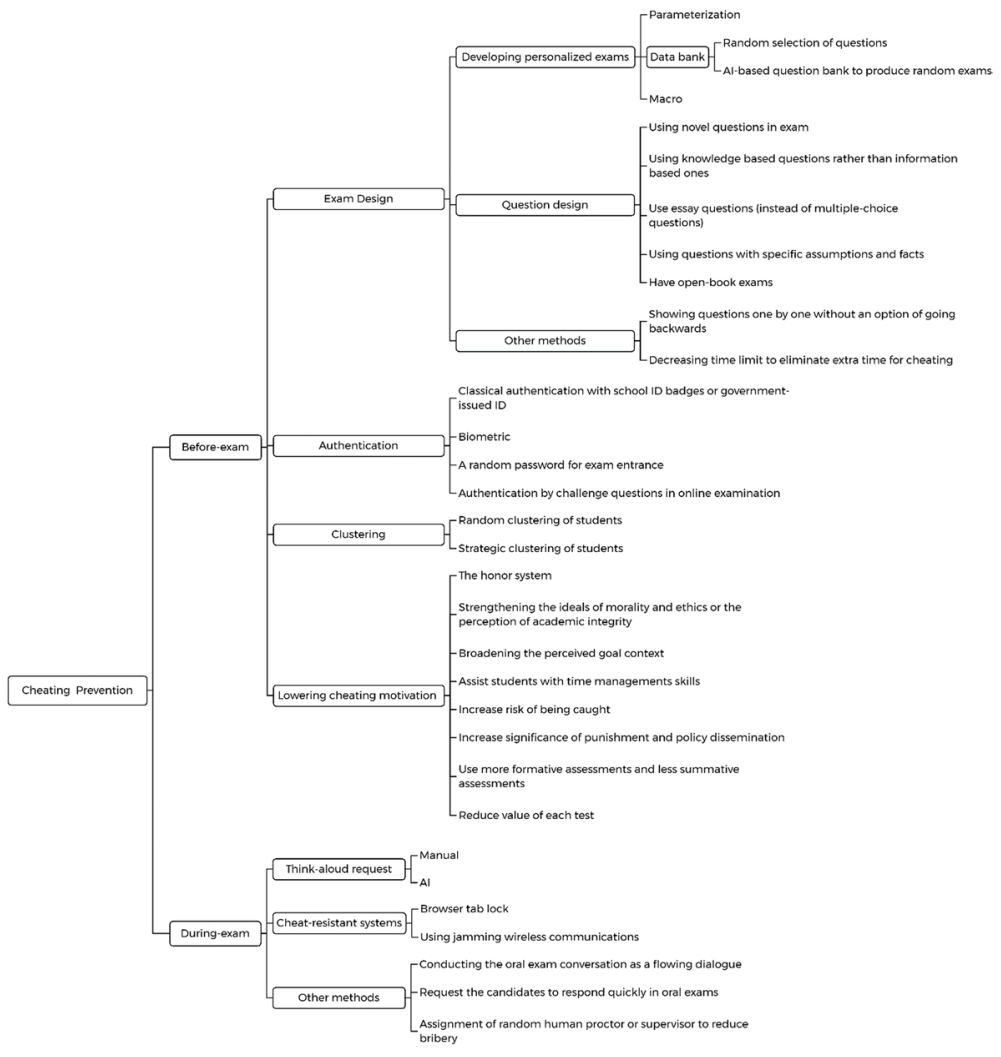

Fig. 12 Cheating prevention

trap websites are incorrect, and consequently, dishonest students could be detected (Korman, 2010). However, this method contradicts professional ethics.

In addition, the teacher can search the internet by hand periodically and try to find all possible web pages that provide solutions matching the exam questions. This approach could be applied to create a pool of potential solutions from the internet that will be used for plagiarism detection purposes after the exam (Norris, 2019).

\subsection{Cheating prevention}

After discussing and analyzing the examinees' motivations for cheating and the reasons which directly or indirectly drive them to commit unethical actions during online examinations, a great deal of concern is gathered around how to decrease cheating in online exams and lower the probability of these actions taking place.

We categorized cheating prevention into two major types, namely, before-exam prevention and during-exam prevention. Figure 12 displays the classification of the cheating prevention methods. 


\subsubsection{Before-exam prevention}

To prevent examinees from cheating, there exist several methods that should be implemented before the exam is held. Each will be discussed in detail as follows.

Exam design In any situation that prevention is concerned, a proven and low-cost approach is a "cheat-resistant" design -A design that inherently prevents some specific cheating types from happening. This is why exam design is so critical. A cheat-resistant exam design, by its nature, prevents a range of possible forms of cheatings from occurring.

One way of achieving a good design is developing personalized exams for each candidate separately. There are several ways to do so, such as parameterization (Manoharan, 2019), which is a set of fixed questions with variable assumption values, using data banks with a large pool of questions to select questions randomly (Manoharan, 2019), (Norris, 2019) or implementing an AI-based method to produce unique exams (Chua \& Lumapas, 2019).

$\mathrm{Li}$ et al. (2020) has put effort into designing a method for randomizing the question orders for each candidate. Their general idea is to show the questions one by one, and besides that, each student gets a different question at a time. This research mathematically proves that examinees cannot get much cheating gain.

In (Manoharan, 2019), the author has investigated an approach to personalizing multiple-choice examinations using the macro. Macro is a computer program fragment that stores data. It has a set of particular inputs for generating random exams based on a question bank. This method could bring freedom and flexibility to the exam design, but it needs basic programming skills.

Another aspect of exam design concentrates specifically on question design. Some of the most valuable methods are listed below.

- Using novel questions: This type of question design is so unique in design and phrasing that it becomes very challenging to be plagiarized even with searching the web (Nguyen et al., 2020).

- Using knowledge-based questions instead of information-based questions: These questions challenge the level of knowledge. The answers are not on the web or in reference books, and they need critical thinking and reasoning (Nguyen et al., 2020).

- Using essay questions rather than multiple-choice questions: During an online exam, multiple-choice questions are highly susceptible to cheating. Hence, long essay questions are preferred (Varble, 2014).

- Using questions with specific assumptions and facts: Although giving extra and not useful facts may mislead any candidate, even those taking the exam honestly, it will reduce the possibility of web-based plagiarism considerably by making it less straightforward to search online (Nguyen et al., 2020).

- Having an open-book exam: Open-book exam questions should test students' understanding, critical reasoning, and analytical skills. Since the answers to these questions are not found in any sources directly, open-book exams may reduce the cheating opportunity (Varble, 2014), (Backman, 2019). 
Finally, other methods not placed into the above categories are mentioned below.

Showing questions one by one without the option of going backward is effective in cheating prevention. If it is employed besides strict time limitations and random question series, collaborative cheating will become quite challenging (Chirumamilla \& Sindre, 2019), (Backman, 2019). By setting strict time limitations, the students do not have enough time to handle cheating, therefore, exam cheating efforts are reduced (Backman, 2019).

Cluskey et al. (2011), emphasize low-cost approaches for addressing online exam cheating. They introduce online exam control procedures (OECP) to achieve this target. Taking the exam only at a defined time and avoiding postponing it for any reason, or changing at least one-third of the questions in the next exam, are some instances of these procedures.

Authentication Authentication is mainly for impersonation prevention before examinations. It could be done classically by checking the school ID badges or government-issued ID by the webcam (Moten et al., 2013) or by a more modern approach like biometrics through fingerprint, palm vein scan (Korman, 2010), eye vein scan (Kigwana \& Venter, 2016), voice, and keystroke biometrics (Norris, 2019).

An interesting method to prevent cheating has been presented in (Moten et al., 2013). Students should call the instructor at a predetermined time to get the password. After the students' voices are recognized by the instructor, they are authenticated and receive a random password for exam entrance. The password is valid until the end of the exam time limit, thus this method makes cheating more difficult (Moten et al., 2013).

The last method of authentication is the one discussed in (Norris, 2019) which uses challenge questions. These are the questions only the student will know, for instance, student ID or personal information. In (Ullah, 2016), an approach is proposed that creates and consolidates a student's profile during the learning process. This information is collected in the form of questions and answers. The questions are pre-defined or extracted from a student's learning activities. A subset of questions is used for authentication, and the students should answer these questions correctly to get access to the online examination. This approach ensures that the person taking the exam is the same one who has completed the course.

Clustering Clustering means partitioning students into several groups based on a predefined similarity measure. In (Topîrceanu, 2017), random and strategic clustering methods are proposed to break friendships during the exam, as cheating prevention techniques. The advantages of random clustering are time and cost efficiencies; however, it is imprecise, and some clusters may include unbroken friendships.

Breaking friendships through clustering relies on two hypotheses (Topîrceanu, 2017):

- Students tend to communicate and cheat with the people they know and feel close to. 
- An individuals' relationship with others on social networks is closely related to their real-life relationships with people.

Regarding the second hypothesis, social network analysis could find students' close friends and people they know. After clustering students, a unique set of exam questions are prepared for each cluster. Consequently, the collaboration of friends to cheat during the online exam becomes challenging.

Lowering cheating motivation Approaches expressed in this section are based on mental and psychological aspects driving students toward academic misbehaviors, and the work being done to reduce these behaviors through controlling mental drivers.

There are several tactics to develop students' moral beliefs encouraging them to avoid unethical behaviors. For instance, implementing honor systems helps build a healthy and ethical environment (Korman, 2010). Another tactic is clarifying academic integrity and morality ideals through establishing educational integrity programs (Korman, 2010).

As Korman (2010) further investigated, changing the students' perception about the goal of studying, could decrease cheating. This could be done by reminding them why learning matters and how it affects their future success. In (Varble, 2014), it is stated that emphasizing the actual value of education will lead to the same result.

Varble (2014), indicates that by improving students' skills such as time management skills, their academic performance will be highly enhanced; accordingly, their academic misbehaviors will be declined. The risks of being caught and the significance of punishments, are inversely related to students' motivation for cheating.

Varble (2014) also mentions that applying formative assessment rather than summative assessment effectively reduces examinees' desire for cheating due to improving their learning outcomes. Formative assessments aim to enhance the candidates' learning performance rather than testing them. On the other hand, summative assessments mostly care about measuring candidates' knowledge and are used to check if they are eligible to pass the course or not.

As an additional description about getting a formative assessment to work, Nguyen et al., (2020) mention that increasing the exam frequency forces students to study course materials repeatedly, resulting in longer retention of information and knowledge in students' minds. This brings about alleviating candidates' motivation for cheating (Nguyen et al., 2020). Varble (2014), also suggests that reducing the value of each test lowers the reward gained by the cheaters over each test; consequently, the motivation for cheating is declined.

A cost-efficient and effective method to lower cheating motivation is to declare the cheating policy for examinees before the exam starts (Moten et al., 2013). Warning students of the consequences of being caught makes them nervous and can significantly decrease cheating. It is necessary to have a confirmation button, so that no excuses can be made by cheaters after the exam. It is such effective that in two experiments, it decreased the number of cheatings by $50 \%$ (CorriganGibbs et al., 2015). It is worth mentioning that in the online environment, having 
an honor system is much less effective than warning about the consequences of cheating if being caught (Fontaine et al., 2020).

\subsubsection{During-exam prevention}

Most cheating prevention methods were discussed in the before-exam section; still, there exist some during-exam prevention tactics, which are presented in this sub-section.

Think-aloud request A rarely mentioned method called Think-aloud request was discussed in (Chirumamilla \& Sindre, 2019). In this method, a request is sent to the student to think aloud about a specific subject (or current question) at random times during the exam. The student has to respond to the request orally, and the voice is recorded for further investigation and cheating detection (e.g., slow response and voice impersonation detection). This mechanism forces students to continuously be ready for responding, which reduces the chance of student cheating. The authors have also mentioned that this system and its questions could be implemented by an AI agent.

Cheat-resistant systems Using cheat-resistant systems will inherently prevent some kinds of cheatings, although they are costly to be implemented (Korman, 2010). Using a browser tab locker (Chua \& Lumapas, 2019) is one of them that prevents unauthorized movements and also identifies them by sniffing their network packets. Another method is using wireless jammers (Chirumamilla \& Sindre, 2019) to disrupt any radio signals (Internet) in an area which usually is the examination hall, during semi-online exams.

Other methods In (Chirumamilla \& Sindre, 2019), some valuable suggestions are given for oral exams. One is conducting the oral exam as a flow of short questions and answers, instead of a long initial question and an extended answer afterward. This is because a flowing dialogue significantly reduces the chance of the examinee following someone else's cues of the solution. They have also suggested that asking the examinee to respond quickly, will facilitate achieving this goal. Besides that, if candidates delay, they may be known suspicious. If a candidate was detected suspicious by the instructor, it is good to interrupt the current question with a new question. This will neutralize the effort made by a third party to help the candidate answer the question.

Another suggestion presented in (Chirumamilla \& Sindre, 2019), is to prepare a big pool of questions for oral exams to prevent questions repetition. As a result, the candidates cannot adjust themselves to the questions asked from previous candidates.

Bribery is a kind of organizational cheating. In (Kigwana \& Venter, 2016) it is indicated that by assigning a random human proctor for the exam right before it started, bribery and beforehand contractions between examinee and proctor would be impossible. 


\section{Discussion}

There is no doubt that online education has changed significantly in recent years. One of the main challenges in online education is the validity of the assessment. Specifically, during the COVID19 pandemic, the integrity of online examinations has become a significant concern. Cheating detection and prevention are hot topics in online assessments. In addition, it is needed to conduct more research on cheating motivation and cheating types. In this research, we review and classify online exam cheating comprehensively.

In this review, only publications written in English were investigated. This could result in review bias, however, it is too difficult and infeasible to review studies in all languages. Many systematic mapping researches consider only publications in English, such as (Nikou \& Economides, 2018) (Martin et al., 2020) (Noorbehbahani et al., 2019) (Wei et al., 2021).

Figure 3 indicates that the publications trend is decreasing, contrary to the hypothesis that online learning is rising, especially with the emergence of the COVID-19. Notably, in this study, online cheating researches have been reviewed. So, Fig. 3 specifically corresponds to online cheating publications not online learning studies in general. However, more investigations of online cheating studies from February 2021 onwards are required to further analyzing the trends.

Several reviewed studies have made no distinction between cheating detection and prevention (Bawarith, 2017; Bawarith et al., 2017; Korman, 2010; Tiong \& Lee, 2021). They employed detection methods to identify dishonest behaviors. Then preventive actions such as making an alarm to the student, or closing the browser tab are performed to deter student cheating. Regarding this definition of prevention, several studies have applied these terms interchangeably, confusing the reader. In this study, we define cheating prevention as strategies and methods that try to prevent the occurrence of cheating in online exams. Considering the latter definition, we attempted to provide a better review and clearer classification to the readers.

One limitation in this domain is the lack of statistics on the popularity of the types, methods, and tools. In (Sabbah, 2017), the most common cheating behaviors and their average risks have been discussed; however, the results are limited to 10 cheating types. Hence, more investigation is required to determine the prevalence of each cheating type and cheating motivation.

An important cheating reason that is overlooked by researchers is learning styles. Students and educators have different preferred learning styles (auditory, visual, kinesthetic and read/write). If teachers and educational institutes don't consider this issue, the course will not be apprehensible for some students, and consequently, they will be motivated to cheat.

Another issue that should be addressed is to evaluate the feasibility of cheating detection and prevention methods. If the equipment for securing online exams is expensive, the students cannot afford it. Therefore, this factor should be considered when developing detection and prevention methods. Cluskey et al. (2011), 
believe that some solutions (e.g., proctors) that detect cheating during online exams are too costly, and their costs outweigh their benefits in some cases. Therefore, cost-effective systems and methods should be implemented.

Privacy and convenience are also vital for examinees. If employed security mechanism for online exams violates privacy and disturbs student convenience, the evaluation will not be practical due to induced stress. Accordingly, these aspects should be considered in cheating detection and prevention systems.

\section{Conclusion}

In this study, cheating in online exams is reviewed and classified comprehensively. It provides the reader with valuable and practical insights to address online exam cheating. To mitigate students cheating, first, it is necessary to know cheating motivations and cheating types and technologies. Furthermore, cheating detection and prevention methods are needed to combat forbidden actions. Detection methods without applying prevention methods could not be effective. As cheating detection and prevention methods are evolved, new cheating types and technologies emerge as well. Consequently, no system can mitigate all kinds of cheating in online exams, and more advanced methods should be employed. It seems the most efficient strategy for cheating handling is to lower cheating motivation.

It should be mentioned that we have not covered studies related to technical attacks and intrusions to online exam systems and teacher devices. This topic could be considered for conducting another review study.

The impact of COVID-19 on online learning and cheating in online exams could be analyzed in future work.

Another future work is to explore how ignoring students' learning styles in teaching and assessment could affect cheating motivation.

Privacy issues, user convenience, and enforced costs of cheating detection and prevention technologies need to be examined in other studies.

In this study, publications from 2010 to 2021 have been reviewed. More investigations are required to review accepted but unpublished studies and publications in 2022 . 


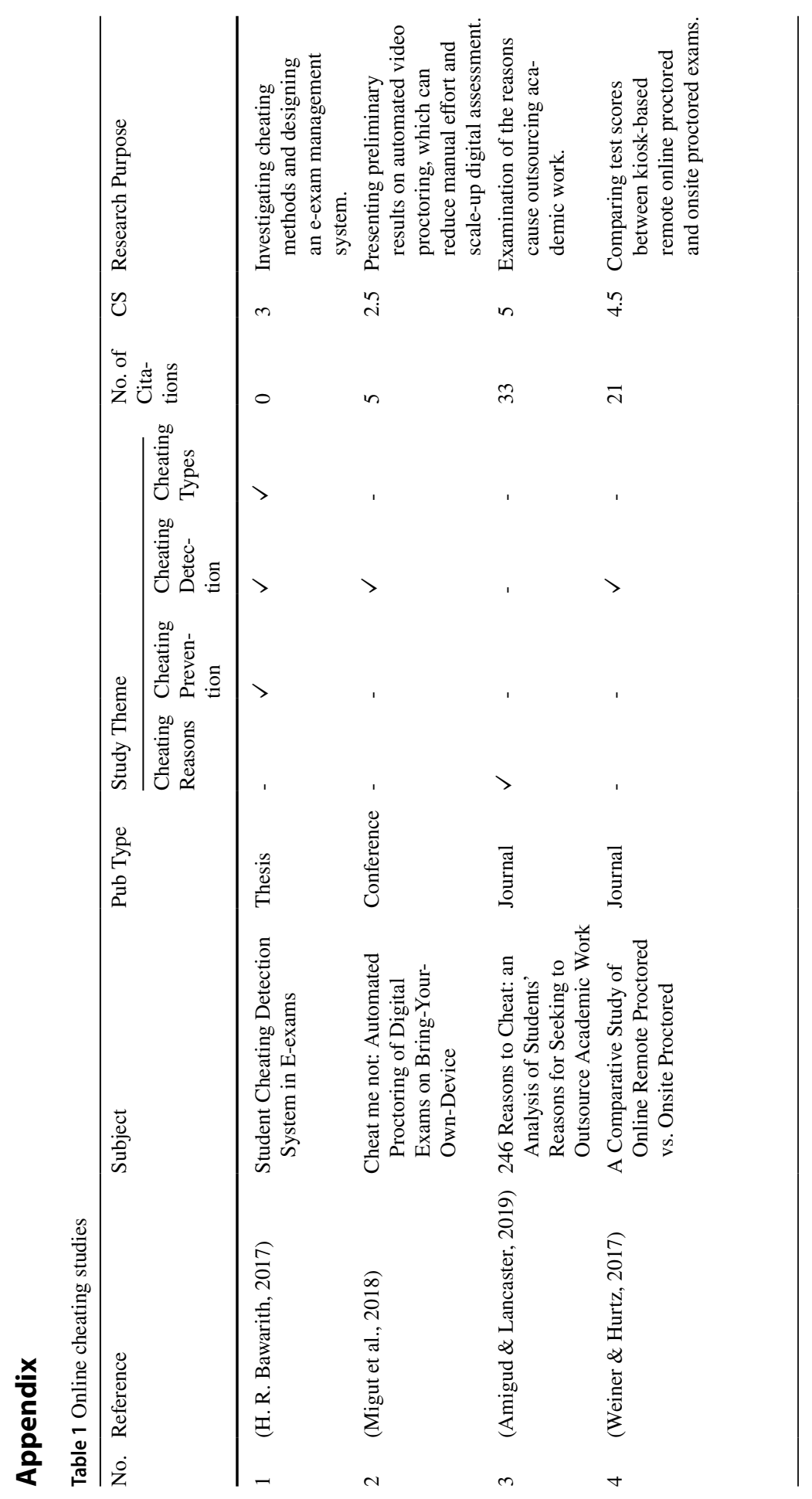




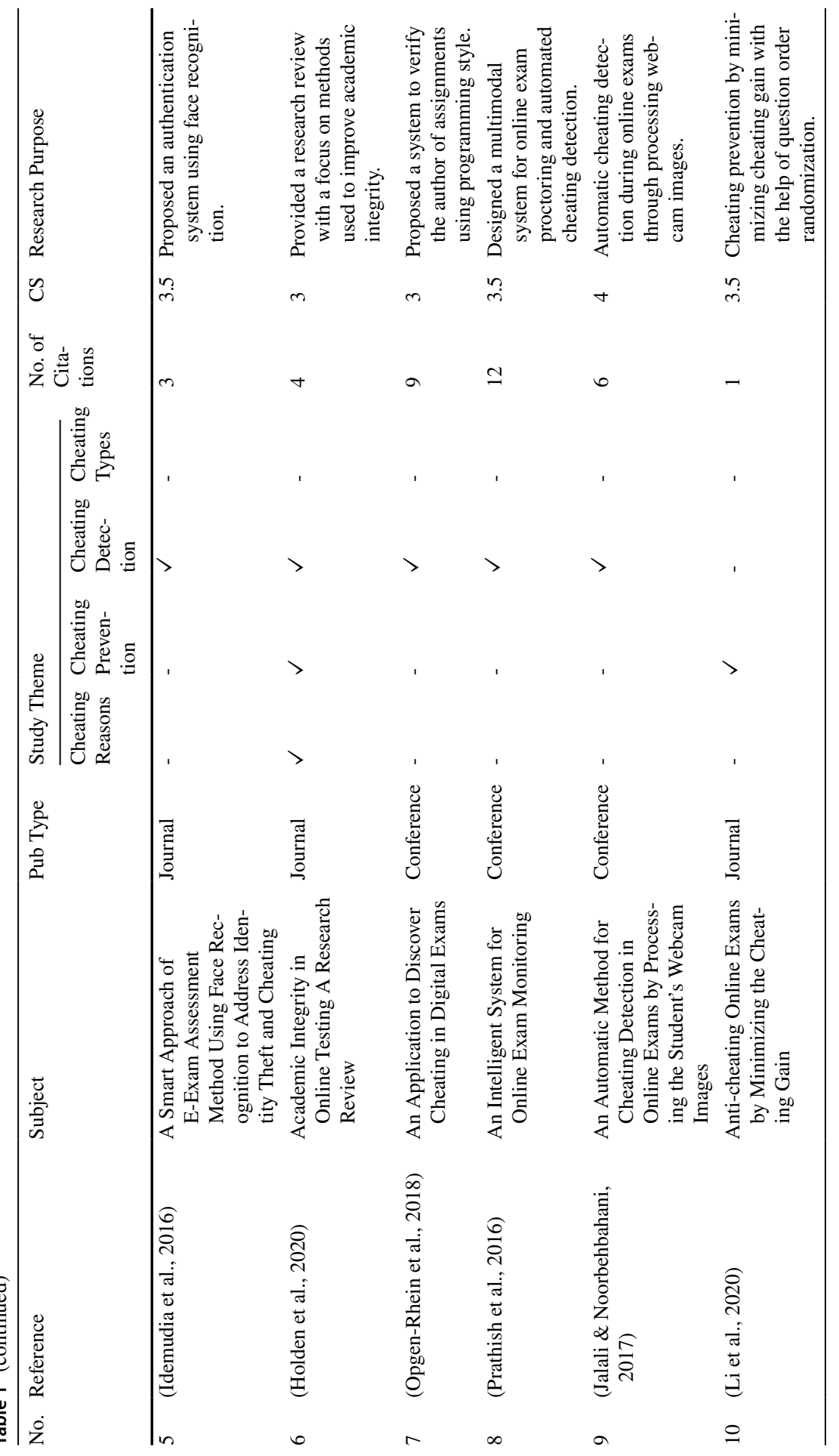




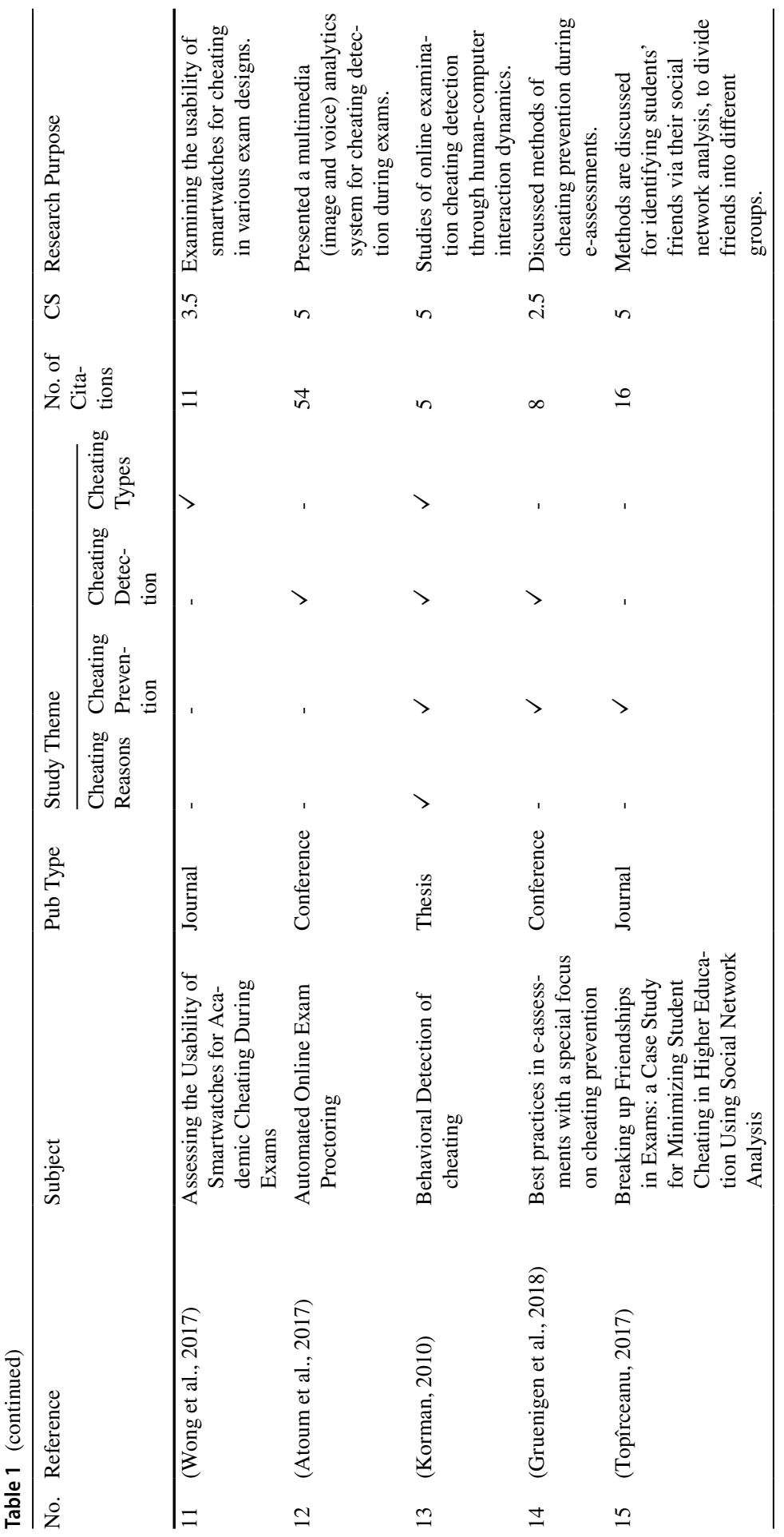




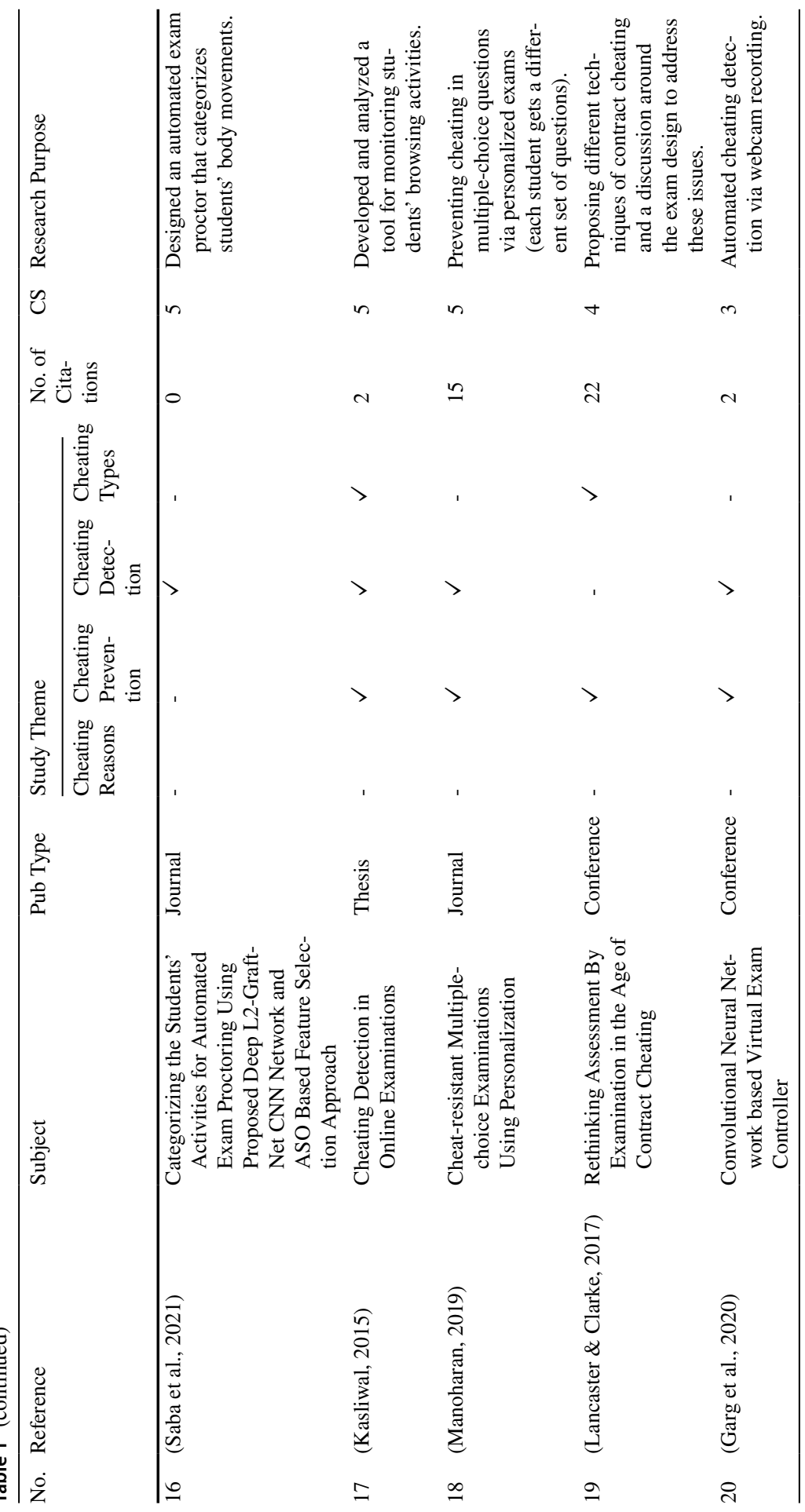




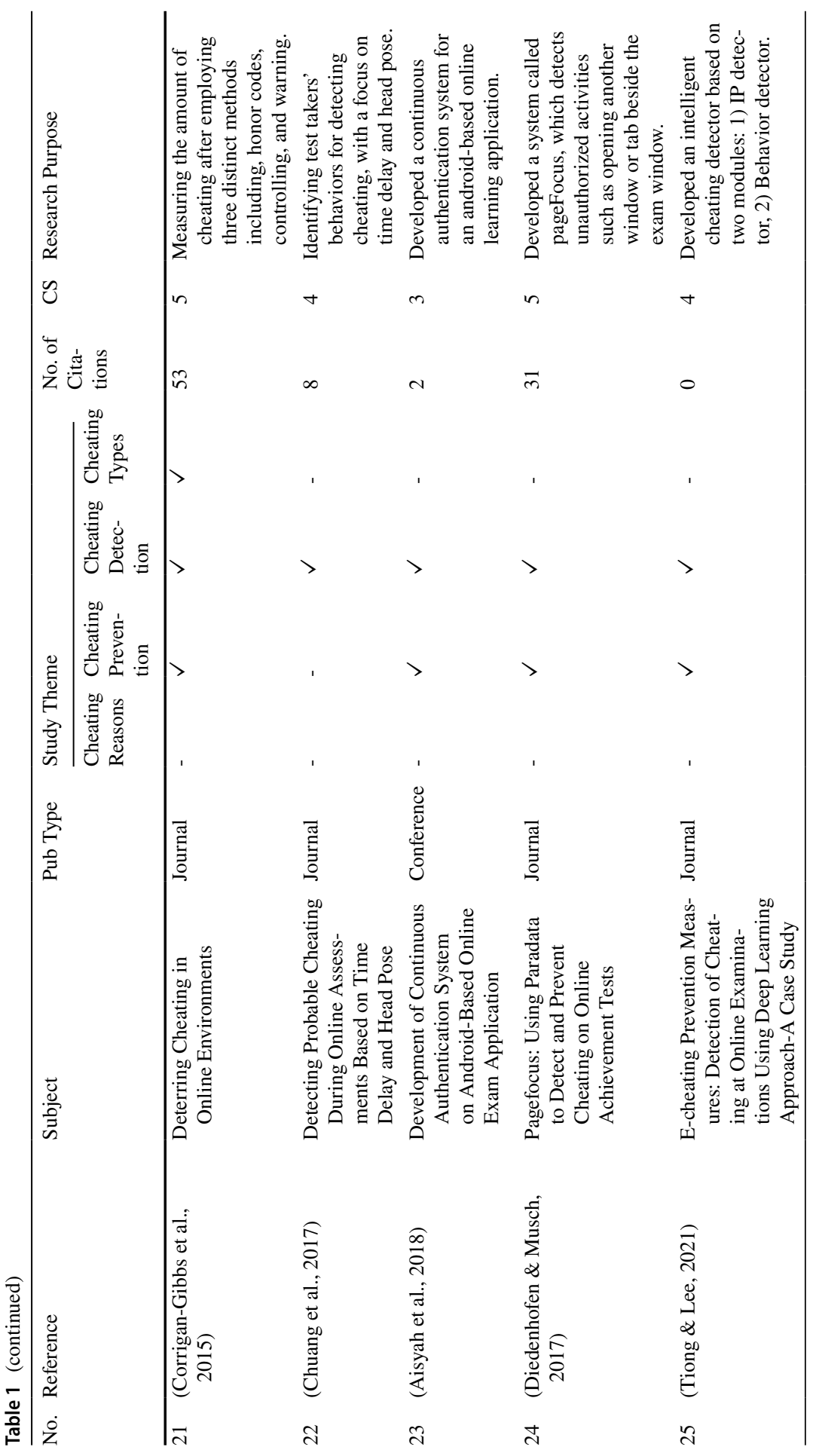




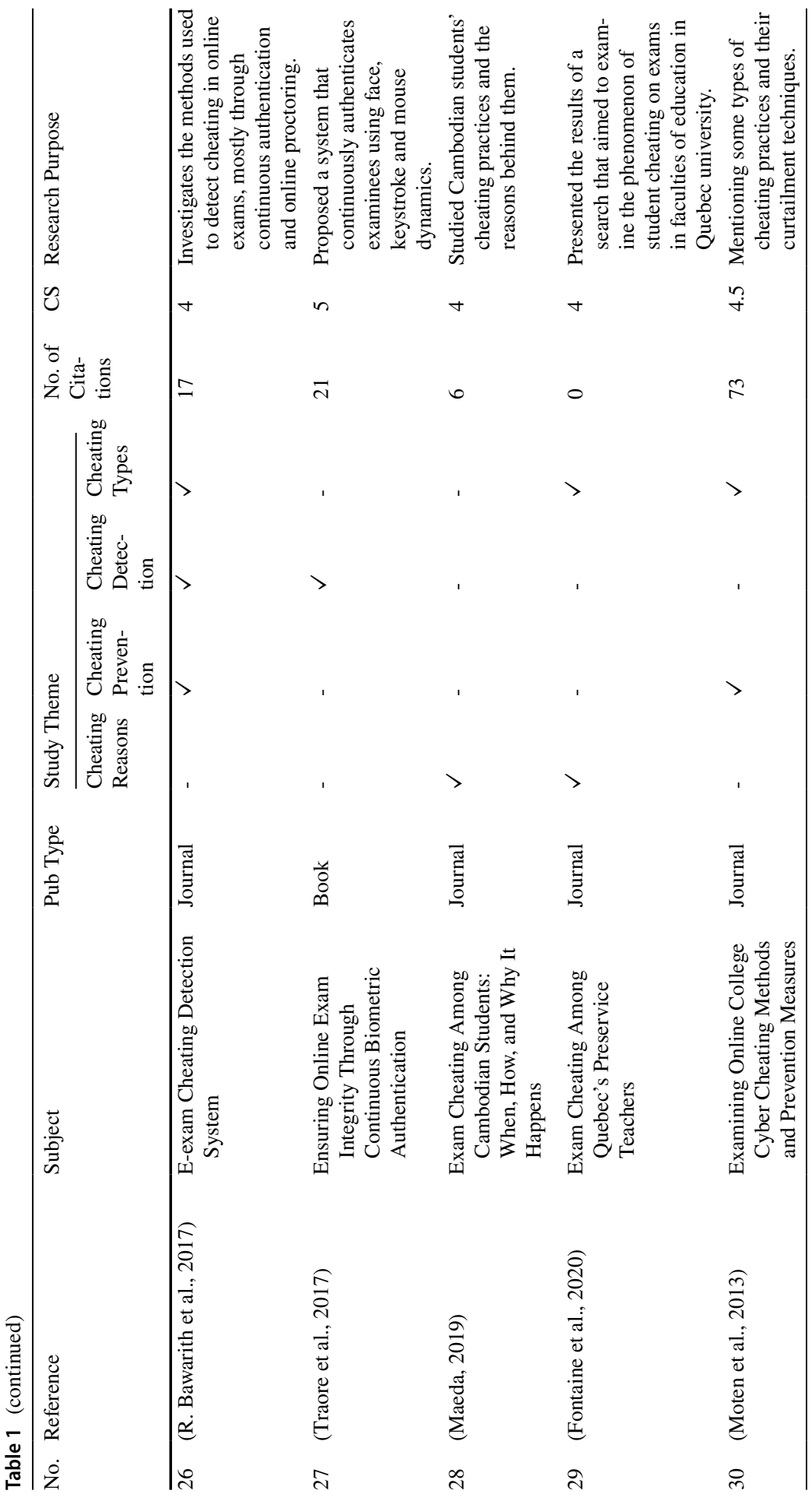




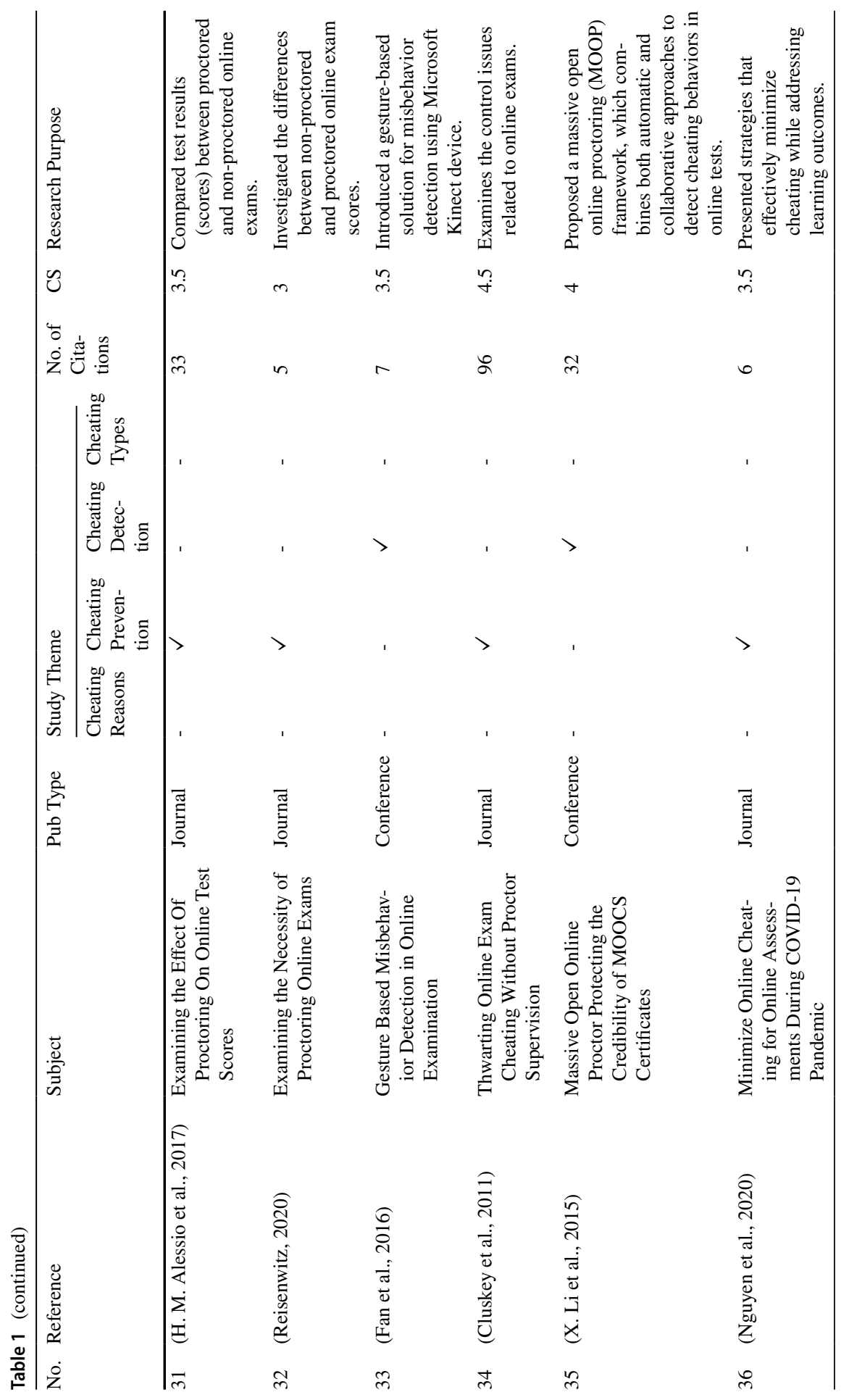




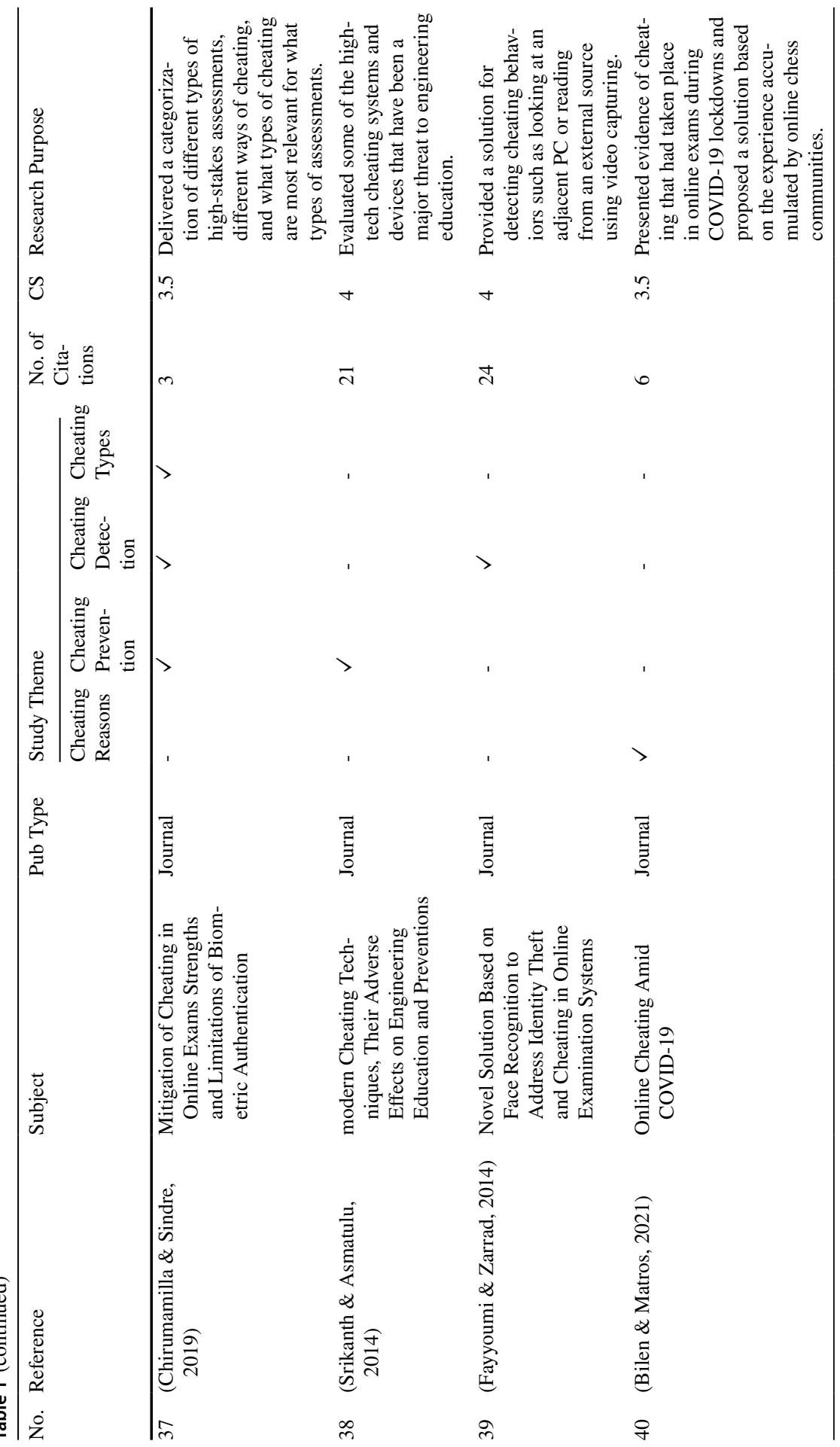




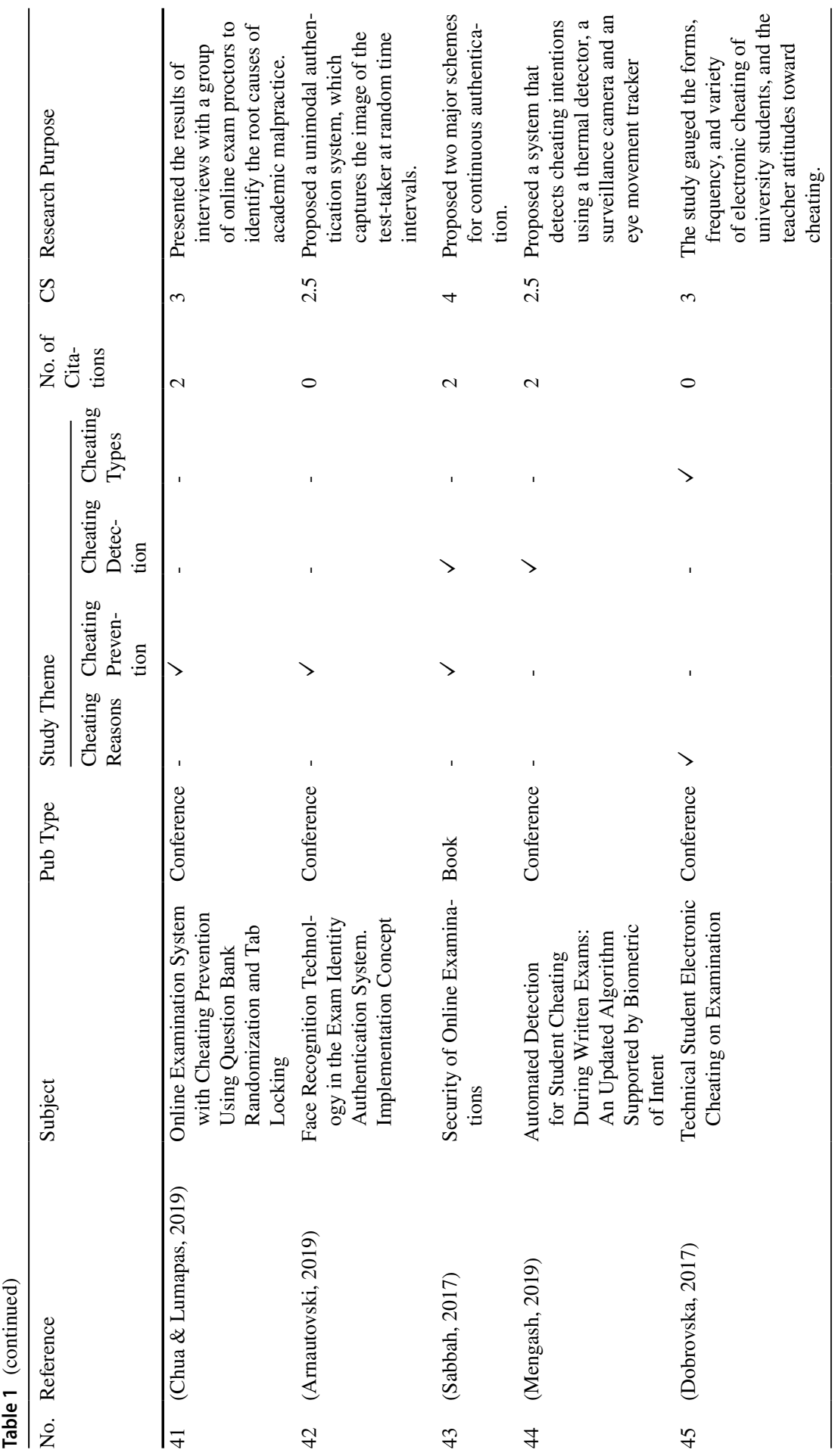




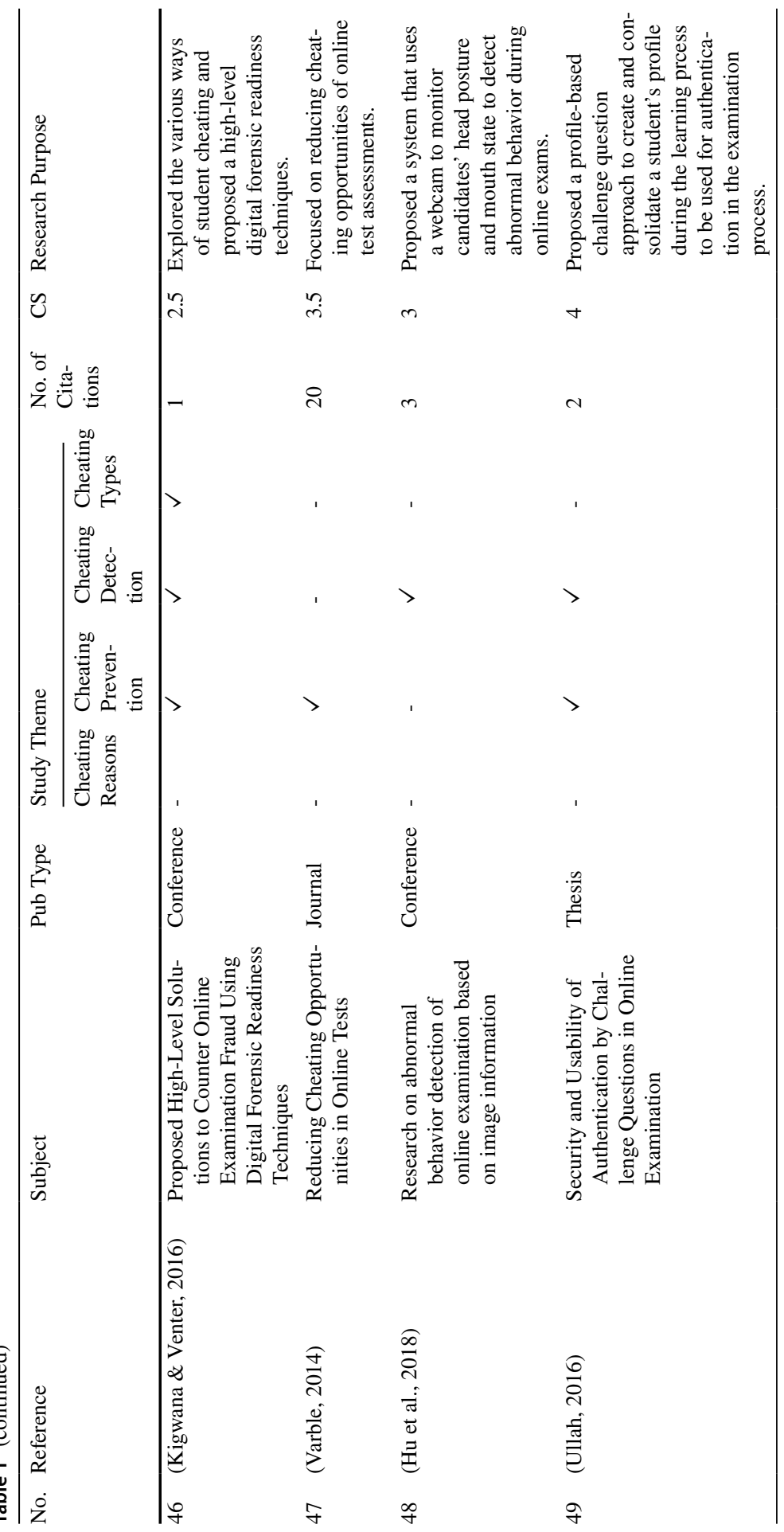




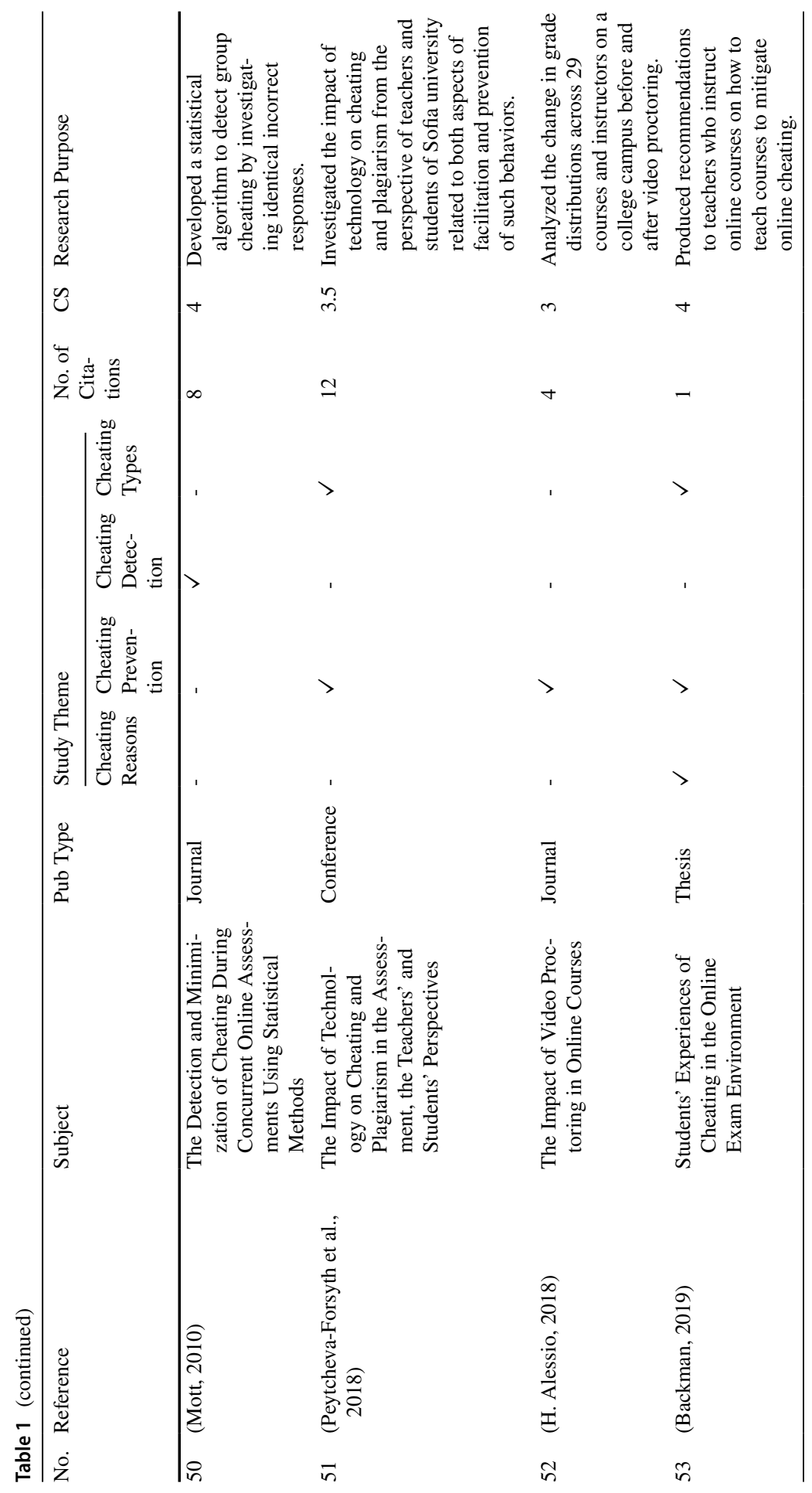




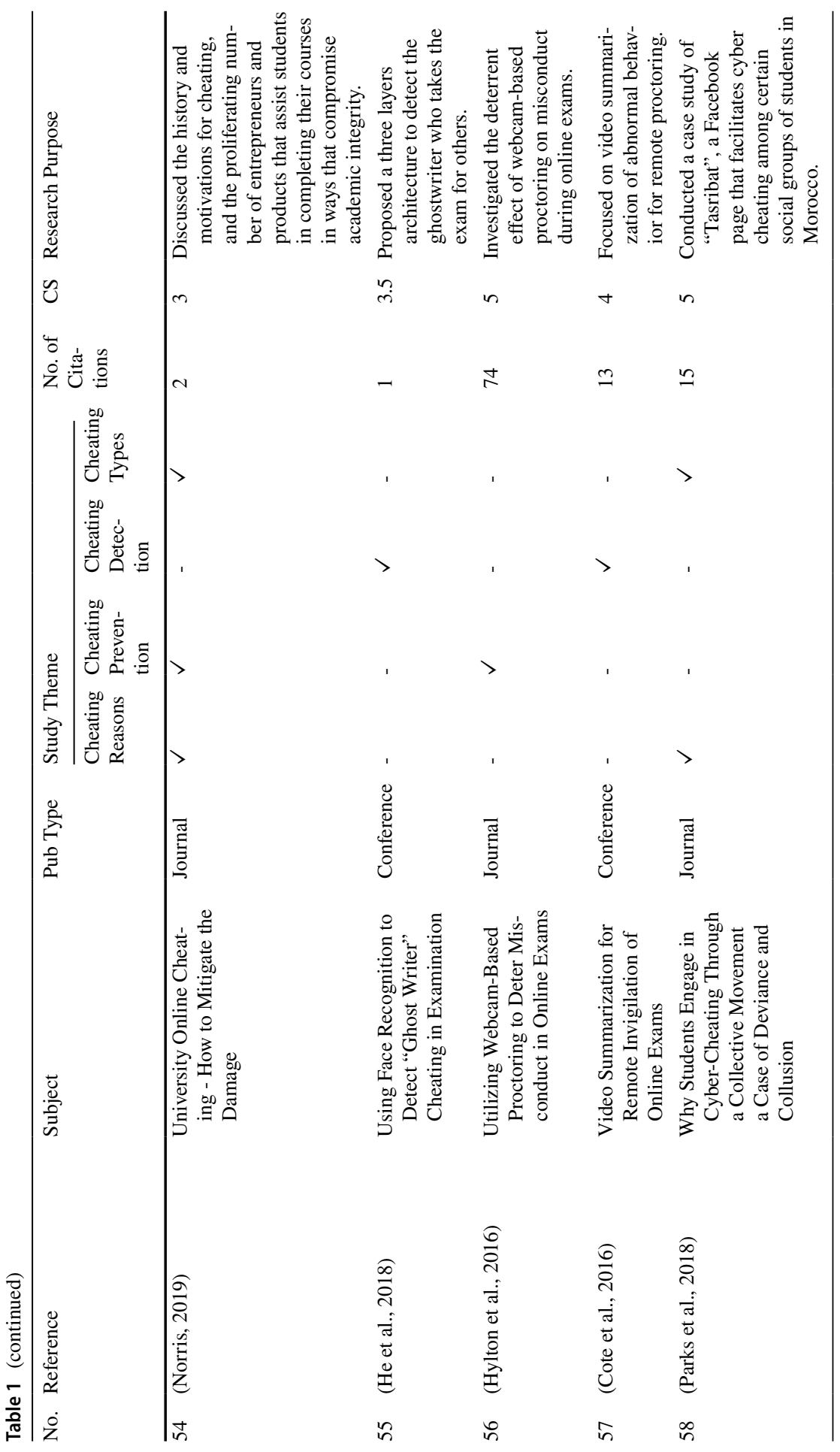




\section{Declarations}

Conflict of interests/Competing interests The authors declare that they have no competing interests.

\section{References}

Aisyah, S., Bandung, Y., \& Subekti, L. B. (2018). Development of Continuous Authentication System on Android-Based Online Exam Application. In 2018 International Conference on Information Technology Systems and Innovation, ICITSI 2018 (pp. 171-176). Padang, Indonesia: IEEE. https://doi. org/10.1109/ICITSI.2018.8695954

Alessio, H. (2018). The Impact of Video Proctoring in Online Courses. Journal on Excellence in ColLege Teaching, 29(3), 1-10.

Alessio, H. M., Malay, N., Maurer, K., Bailer, A. J., \& Rubin, B. (2017). Examining the Effect of Proctoring on Online Test Scores. Online Learning, 2013(1), 1-16.

Amigud, A., \& Lancaster, T. (2019). 246 reasons to cheat: An analysis of students' reasons for seeking to outsource academic work. Computers and Education, 134, 98-107. https://doi.org/10.1016/j.compe du.2019.01.017

Arnautovski, L. (2019). Face recognition technology in the exam identity authentication system - implementation concept. In 2nd International Scientific Conference MILCON'19 (pp. 51-56). Olsztyn, Poland.

Atoum, Y., Chen, L., Liu, A. X., Hsu, S. D. H., \& Liu, X. (2017). Automated Online Exam Proctoring. IEEE Transactions on Multimedia, 19(7), 1609-1624. https://doi.org/10.1109/TMM.2017.2656064

Backman, J. (2019). Student s' Experiences of Cheating in the Online Exam Environment.

Bawarith, H. R. (2017). Student Cheating Detection System in E-exams. KING ABDULAZIZ UNIVERSITY.

Bawarith, R., Basuhail, A., Fattouh, A., \& Gamalel-din, P. S. (2017). E-exam Cheating Detection System. International Journal of Advanced Computer Science and Applications, 8(4), 176-181.

Bilen, E., \& Matros, A. (2021). Online Cheating Amid COVID-19. Journal of Economic Behavior \& Organization, 182, 196-211.

Chirumamilla, A., \& Sindre, G. (2019). Mitigation of Cheating in Online Exams: Strengths and Limitations of. In Biometric Authentication in Online Learning Environments (pp. 47-68). IGI Global. https://doi.org/10.4018/978-1-5225-7724-9.ch003

Chua, S. S., \& Lumapas, Z. R. (2019). Online Examination System with Cheating Prevention Using Question Bank Randomization and Tab Locking. 2019 4th International Conference on Information Technology (InCIT), 126-131.

Chuang, C. Y., Craig, S. D., \& Femiani, J. (2017). Detecting probable cheating during online assessments based on time delay and head pose. Higher Education Research and Development, 36(6), 1123-1137. https://doi.org/10.1080/07294360.2017.1303456

Cluskey, G. R. C. Jr, Ehlen, C. R., \& Raiborn, M. H. (2011). Thwarting Online Exam Cheating without Proctor Supervision, 4, 1-7.

Corrigan-Gibbs, H., Gupta, N., Northcutt, C., Cutrell, E., \& Thies, W. (2015). Deterring cheating in online environments. ACM Transactions on Computer-Human Interaction, 22(6). https://doi.org/10. $1145 / 2810239$

Cote, M., Jean, F., Albu, A. B., \& Capson, D. (2016). Video Summarization for Remote Invigilation of Online Exams. In 2016 IEEE Winter Conference on Applications of Computer Vision (pp. 1-9). NY, USA.

Curran, K., Middleton, G., \& Doherty, C. (2011). Cheating in Exams with Technology. International Journal of Cyber Ethics in Education, 1(2), 54-62. https://doi.org/10.4018/ijcee.2011040105

Dendir, S., \& Maxwell, R. S. (2020). Cheating in online courses: Evidence from online proctoring. Computers in Human Behavior Reports, 2, 100033. https://doi.org/10.1016/j.chbr.2020.100033

Diedenhofen, B., \& Musch, J. (2017). PageFocus: Using paradata to detect and prevent cheating on online achievement tests. Behavior Research Methods, 49(4), 1444-1459. https://doi.org/10.3758/ s13428-016-0800-7 
Dobrovska, D. (2017). Technical Student Electronic Cheating on Examination. In M. E. Auer, D. Guralnick, \& J. Uhomoibhi (Eds.), Interactive Collaborative Learning (pp. 525-531). Springer International Publishing.

Fan, Z., Xu, J., Liu, W., \& Cheng, W. (2016). Gesture based Misbehavior Detection in Online Examination. In The 11th International Conference on Computer Science \& Education (pp. 234-238). NagoyaF, Japan.

Fayyoumi, A., \& Zarrad, A. (2014). Novel Solution Based on Face Recognition to Address Identity Theft and Cheating in Online Examination Systems. Advances in Internet of Things, 4(April), 5-12.

Fluck, A. E. (2019). An international review of eExam technologies and impact. Computers \& Education, 132, 1-15. https://doi.org/10.1016/j.compedu.2018.12.008

Fontaine, S., Frenette, E., \& Hébert, M. (2020). Exam cheating among Quebec's preservice teachers : the influencing factors. International Journal for Educational Integrity, 16(14), 1-18.

Garg, K., Verma, K., Patidar, K., Tejra, N., \& Petidar, K. (2020). Convolutional Neural Network based Virtual Exam Controller. In Proceedings of the International Conference on Intelligent Computing and Control Systems, ICICCS 2020 (pp. 895-899). Secunderabad, India. https://doi.org/10.1109/ ICICCS48265.2020.9120966

Gruenigen, D. Von, de Azevedo e Souza, F. B., Pradarelli, B., Magid, A., \& Cieliebak, M. (2018). Best practices in e-assessments with a special focus on cheating prevention. In 2018 \{IEEE\} Global Engineering Education Conference, \{EDUCON\} 2018, Santa Cruz de Tenerife, Tenerife, Islas Canarias, Spain, April 17-20, 2018 (pp. 893-899). IEEE. https://doi.org/10.1109/EDUCON.2018.8363325

He, H., Zheng, Q., Li, R., \& Dong, B. (2018). Using Face Recognition to Detect “ Ghost Writer " Cheating in Examination. In Edutainment, Lecture Notes in Computer Science (Vol. 11462, pp. 389-397). Springer International Publishing. https://doi.org/10.1007/978-3-030-23712-7

Holden, O., Kuhlmeier, V., \& Norris, M. (2020). Academic Integrity in Online Testing: A Research Review. https://doi.org/10.31234/osf.io/rjk7g

Hu, S., Jia, X., \& Fu, Y. (2018). Research on Abnormal Behavior Detection of Online Examination Based on Image Information. In 10th International Conference on Intelligent Human-Machine Systems and Cybernetics (IHMSC) (Vol. 02, pp. 88-91). Hangzhou, China: IEEE. https://doi.org/10.1109/ IHMSC.2018.10127

Hylton, K., Levy, Y., \& Dringus, L. P. (2016). Computers \& Education Utilizing webcam-based proctoring to deter misconduct in online exams. Computers \& Education, 92-93, 53-63. https://doi.org/10. 1016/j.compedu.2015.10.002

Idemudia, S., Rohani, M. F., Siraj, M., \& Othman, S. H. (2016). A Smart Approach of E-Exam Assessment Method Using Face Recognition to Address Identity Theft and Cheating. International Journal of Computer Science and Information Security, 14(10), 515-522. Retrieved from https://sites. google.com/site/ijcsis/

Jalali, K., \& Noorbehbahani, F. (2017). An Automatic Method for Cheating Detection in Online Exams by Processing the Students Webcam Images. In 3rd Conference on Electrical and Computer Engineering Technology (E-Tech 2017), Tehran, Iran (pp. 1-6). Tehran, Iran.

Kasliwal, G. (2015). Cheating Detection in Online Examinations.

Kigwana, I., \& Venter, H. (2016). Proposed high-level solutions to counter online examination fraud using digital forensic readiness techniques. Proceedings of the 11th International Conference on Cyber Warfare and Security, ICCWS 2016, 407-414.

Korman, M. (2010). Behavioral detection of cheating in online examination. Retrieved from https://pure. ltu.se/ws/files/31188849/LTU-DUPP-10112-SE.pdf

Lancaster, T., \& Clarke, R. (2017). Rethinking Assessment By Examination in the Age of Contract Cheating. Plagiarism Across Europe and Beyond 2017.

Li, M., Sikdar, S., Xia, L., \& Wang, G. (2020). Anti-cheating Online Exams by Minimizing the Cheating Gain, (May). https://doi.org/10.20944/preprints202005.0502.v1

Li, X., Yueran, K. C., \& Alexander, Y. (2015). Massive Open Online Proctor : Protecting the Credibility of MOOCs Certificates, 1129-1137.

Maeda, M. (2019). Exam cheating among Cambodian students : when, how , and why it happens. Compare: A Journal of Comparative and International Education, 1-19. https://doi.org/10.1080/03057 925.2019.1613344

Manoharan, S. (2019). Cheat-resistant multiple-choice examinations using personalization. Computers and Education, 130, 139-151. https://doi.org/10.1016/j.compedu.2018.11.007 
Martin, F., Sun, T., \& Westine, C. D. (2020). A systematic review of research on online teaching and learning from 2009 to 2018. Computers \& Education, 159, 104009. https://doi.org/10.1016/j.compe du.2020.104009

Mengash, H. (2019). Automated Detection for Student Cheating During Written Exams: An Updated Algorithm Supported by Biometric of Intent. In First International Conference on Computing (pp. 303-3111). Riyadh, Saudi Arabia. https://doi.org/10.1007/978-3-030-36368-0

Migut, G., Koelma, D., Snoek, C. G., \& Brouwer, N. (2018). Cheat Me Not: Automated Proctoring Of Digital Exams On Bring-Your-Own-Device. In The 23rd Annual ACM Conference On In- novation And Technology In Computer Science Education (p. 388). New York, NY, USA.

Moten, J. M. Jr, Fitterer, A., Brazier, E., Leonard, J., Brown, A., \& Texas, A. (2013). Examining Online College Cyber Cheating Methods and Prevention Measures. Electronic Journal of E-Learning, 11(2), 139-146.

Mott, J. H. (2010). The Detection and Minimization of Cheating During Concurrent Online Assessments Using Statistical Methods. Collegiate Aviation Review, 28(2), 32-46.

Nguyen, J. G., Keuseman, K. J., \& Humston, J. J. (2020). Minimize Online Cheating for Online Assessments During COVID-19 Pandemic. https://doi.org/10.1021/acs.jchemed.0c00790

Nikou, S. A., \& Economides, A. A. (2018). Mobile-based assessment: A literature review of publications in major referred journals from 2009 to 2018. Computers \& Education, 125, 101-119. https://doi. org/10.1016/j.compedu.2018.06.006

Noorbehbahani, F., Salehi, F., \& Jafar Zadeh, R. (2019). A systematic mapping study on gamification applied to e-marketing. Journal of Research in Interactive Marketing, 13(3). https://doi.org/10.1108/ JRIM-08-2018-0103

Norris, M. (2019). University online cheating - how to mitigate the damage. Research in Higher Education Journal, 37, 1-20.

Opgen-Rhein, J., Küppers, B., \& Schroeder, U. (2018). An application to discover cheating in digital exams. In ACM International Conference Proceeding Series. Koli, Finland. https://doi.org/10.1145/ 3279720.3279740

Page, M. J., Moher, D., Bossuyt, P. M., Boutron, I., Hoffmann, T. C., Mulrow, C. D., \& Mckenzie, J. E. (2021). PRISMA 2020 explanation and elaboration: Updated guidance and exemplars for reporting systematic reviews. BMJ, 372,. https://doi.org/10.1136/bmj.n160

Parks, R. F., Lowry, P. B., Wigand, R. T., Agarwal, N., \& Williams, T. L. (2018). Why students engage in cyber-cheating through a collective movement: A case of deviance and collusion. Computers and Education, 125, 308-326. https://doi.org/10.1016/j.compedu.2018.04.003

Peytcheva-Forsyth, R., Aleksieva, L., \& Yovkova, B. (2018). The impact of technology on cheating and plagiarism in the assessment - The teachers' and students' perspectives. In AIP Conference Proceedings 2048 (Vol. 020037, pp. 1-11).

Prathish, S., Athi Narayanan, S., \& Bijlani, K. (2016). An intelligent system for online exam monitoring. In Proceedings - 2016 International Conference on Information Science, ICIS 2016 (pp. 138-143). Dublin, Ireland. https://doi.org/10.1109/INFOSCI.2016.7845315

Reisenwitz, T. H. (2020). Examining the Necessity of Proctoring Online Exams. Journal of Higher Education Theory and Practice, 20(1), 118-124.

Saba, T., Rehman, A., Jamail, N. S. M., Marie-Sainte, S. L., Raza, M., \& Sharif, M. (2021). Categorizing the Students' Activities for Automated Exam Proctoring Using Proposed Deep L2-GraftNet CNN Network and ASO Based Feature Selection Approach. IEEE Access, 9, 47639-47656. https://doi. org/10.1109/ACCESS.2021.3068223

Sabbah, Y. W. (2017). Security of Online Examinations. In Data Analytics and Decision Support for Cybersecurity (pp. 157-200). Springer International Publishing.

Srikanth, M., \& Asmatulu, R. (2014). Modern Cheating Techniques, Their Adverse Effects on Engineering Education and preventions. International Journal of Mechanical Engineering Education, 42(2), 129-140. https://doi.org/10.7227/IJMEE.0005

Tiong, L. C. O., \& Lee, H. J. (2021). E-cheating Prevention Measures: Detection of Cheating at Online Examinations Using Deep Learning Approach -- A Case Study, $X X(\mathrm{Xx}), 1-9$. Retrieved from http:// arxiv.org/abs/2101.09841

Topîrceanu, A. (2017). Breaking up friendships in exams: A case study for minimizing student cheating in higher education using social network analysis. Computers and Education, 115, 171-187. https:// doi.org/10.1016/j.compedu.2017.08.008 
Traore, I., Nakkabi, Y., Saad, S., \& Sayed, B. (2017). Ensuring Online Exam Integrity Through Continuous Biometric Authentication. In Information Security Practices (pp. 73-81). Springer International Publishing. https://doi.org/10.1007/978-3-319-48947-6

Turner, S. W., \& Uludag, S. (2013). Student perceptions of cheating in online and traditional classes. Proceedings - Frontiers in Education Conference, FIE, (October 2013), 1131-1137. https://doi.org/ 10.1109/FIE.2013.6685007

Ullah, A. (2016). Security and Usability of Authentication by Challenge Questions in Online Examination. University of Hertfordshire.

Valverde-Berrocoso, J., Garrido-Arroyo, M. del C., Burgos-Videla, C., \& Morales-Cevallos, M. B. (2020). Trends in Educational Research about e-Learning: A Systematic Literature Review (2009_ 2018). Sustainability, 12(12). https://doi.org/10.3390/su12125153

Varble, D. (2014). Reducing Cheating Opportunities in Online Test Online Tests, 3(3).

Watson, G., \& Sottile, J. (2010). Cheating in the Digital Age: Do Students Cheat More in Online Courses?. Online Journal of Distance Learning Administration, 13(1).

Wei, X., Saab, N., \& Admiraal, W. (2021). Assessment of cognitive, behavioral, and affective learning outcomes in massive open online courses: A systematic literature review. Computers \& Education, 163, 104097. https://doi.org/10.1016/j.compedu.2020.104097

Weiner, J. A., \& Hurtz, G. M. (2017). A comparative Study of Online Remote Proctored Vs Onsite Proctored. Journal of Applied Testing Technology, 18(1), 13-20.

Wong, S., Yang, L., Riecke, B., Cramer, E., \& Neustaedter, C. (2017). Assessing the usability of smartwatches for academic cheating during exams. Proceedings of the 19th International Conference on Human-Computer Interaction with Mobile Devices and Services, MobileHCI 2017. https://doi.org/ $10.1145 / 3098279.3098568$

Xiong, Y., \& Suen, H. K. (2018). Assessment approaches in massive open online courses: Possibilities, challenges and future directions. International Review of Education, 64(2), 241-263. https://doi.org/ $10.1007 / \mathrm{s} 11159-018-9710-5$

Publisher's note Springer Nature remains neutral with regard to jurisdictional claims in published maps and institutional affiliations. 